\title{
A Novel Full-Euler Low Mach Number IMEX Splitting
}

\author{
Jonas Zeifang ${ }^{1, *}$, Jochen Schütz ${ }^{2}$, Klaus Kaiser ${ }^{2,3}$, Andrea Beck ${ }^{1}$, \\ Maria Lukáčová-Medvid'ová ${ }^{4}$ and Sebastian Noelle ${ }^{3}$ \\ ${ }^{1}$ IAG, Universität Stuttgart, Pfaffenwaldring 21, DE-70569 Stuttgart, Germany. \\ ${ }^{2}$ Faculty of Sciences, Hasselt University, Agoralaan Gebouw D, BE-3590 \\ Diepenbeek, Belgium. \\ ${ }^{3}$ IGPM, RWTH Aachen University, Templergraben 55, DE-52062 Aachen, \\ Germany. \\ ${ }^{4}$ Institut für Mathematik, Johannes Gutenberg-Universität Mainz, Staudingerweg 9, \\ DE-55128 Mainz, Germany.
}

Received 15 October 2018; Accepted (in revised version) 23 January 2019

\begin{abstract}
In this paper, we introduce an extension of a splitting method for singularly perturbed equations, the so-called RS-IMEX splitting [Kaiser et al., Journal of Scientific Computing, 70(3), 1390-1407], to deal with the fully compressible Euler equations. The straightforward application of the splitting yields sub-equations that are, due to the occurrence of complex eigenvalues, not hyperbolic. A modification, slightly changing the convective flux, is introduced that overcomes this issue. It is shown that the splitting gives rise to a discretization that respects the low-Mach number limit of the Euler equations; numerical results using finite volume and discontinuous Galerkin schemes show the potential of the discretization.
\end{abstract}

AMS subject classifications: 35L81, 65M08, 65M60, 76M45

Key words: Euler equations, low-Mach, IMEX Runge-Kutta, RS-IMEX.

\section{Introduction}

The modeling of many processes in fluid dynamics requires the solution of the compressible Euler equations. This hyperbolic set of equations contains propagation waves at different speeds. At low Mach numbers, the propagation speeds of the waves differ by

\footnotetext{
*Corresponding author. Email addresses: zeifang@iag.uni-stuttgart.de (J. Zeifang), jochen.schuetz@uhasselt.be (J. Schütz), kaiser@igpm.rwth-aachen.de (K. Kaiser), beck@iag.uni-stuttgart.de (A. Beck), lukacova@uni-mainz.de (M. Lukáčová-Medvid'ová), noelle@igpm.rwth-aachen.de (S. Noelle)
} 
several orders of magnitude leading to a stiff behavior of the equation system. Classical explicit time integration schemes would require the resolution of all waves for stability reasons, even though the contribution of the fast waves on the solution can be negligible for many applications. Fully implicit schemes can overcome this stability issue, but they require specially designed numerical fluxes and need the solution of large nonlinear systems, see e.g. [3,35]. One way to obtain a stable and efficient numerical method is to split the equations into a stiff and a non-stiff part and handle those parts implicitly and explicitly, respectively. Such a procedure leads to IMEX schemes, see, e.g., $[2,6,18]$ and the references therein. This approach is particularly advantageous in cases where the time resolved resolution of the slow waves is of interest. The choice of the splitting is important. It determines such crucial properties as stability, accuracy and efficiency. It is therefore not surprising that many people have worked on identifying suitable splittings for various types of the Euler equations, starting from the groundbreaking work of Klein [26]. To name a few - this list is by no means exhaustive - we refer to $[4,8-11,13,15,22,29,34]$. The present splittings have different drawbacks such as the need for solving an elliptic equation or large nonlinear systems, requiring staggered meshes, being limited to low order discretization or not being able to be applied to the full Euler equations. Recently, a new splitting for the isentropic Euler equations based on the solution of the incompressible Euler equations has been introduced, see [21-23,36,37]. This splitting can be combined with a high order discretization in space and time and remains linear in the implicit part which significantly simplifies the solution of the associated system. Applying it in a straightforward manner to the full Euler equations of gas dynamics results in a scheme where the explicit part is no longer hyperbolic. This is of course an unwanted feature, as the method can become instable, which will be discussed in this work.

The purpose of this paper is to propose a modification of the splitting to overcome this issue, thereby obtaining a stable and accurate scheme for the Euler equations at low Mach numbers. The final splitting is combined with an IMEX Runge-Kutta method and is shown to be asymptotically consistent. Then, spatial discretization is achieved via either a finite volume [33] approach or a discontinuous Galerkin approach [17, 27]. In future it would be interesting to investigate how our methodology can be generalized for further non-standard space approximations of the Euler equations known in the literature, such as the ALE-multi-moment finite volume scheme [19] or high-resolution Lagrangian methods [30].

The paper is structured as follows: In Section 2 we introduce the RS-IMEX splitting for the Euler equations and describe how it can be modified in order to obtain a hyperbolic explicit and implicit system. Following, Section 3 introduces the numerical discretization of the novel splitting. Here, the asymptotic consistency of the semi discrete scheme is proven in Section 3.2 and the spatial discretization is introduced in Section 3.3. The limitations of the splitting are discussed in the subsequent section (Section 4). Following, the low Mach number capabilities of the scheme are illustrated with suitable testcases in Section 5. Finally, the paper is closed with a conclusion and outlook (Section 6). 


\section{A novel splitting for the Euler equations}

\subsection{Governing equations}

In order to analyze the behavior of the numerical scheme at low Mach numbers, we write the compressible Euler equations in non-dimensional form as

$$
\partial_{t}\left(\begin{array}{c}
\rho \\
\rho \boldsymbol{u} \\
E
\end{array}\right)+\nabla \cdot \underbrace{\left(\begin{array}{c}
\rho \boldsymbol{u} \\
\rho \boldsymbol{u} \otimes \boldsymbol{u}+\frac{p}{\varepsilon^{2}} I d \\
\boldsymbol{u}(E+p)
\end{array}\right)}_{=: \boldsymbol{w}}=0, \quad \forall(x, t) \in \Omega \times \mathbb{R}^{+},
$$

where $\rho$ denotes density, $\boldsymbol{u}$ velocity, $E$ energy density and the pressure is computed via the equation of state for a perfect gas

$$
p(\rho, \rho u, E):=(\gamma-1)\left(E-\frac{\varepsilon^{2}}{2} \rho\|\boldsymbol{u}\|_{2}^{2}\right),
$$

with $\gamma$ being the isentropic expansion coefficient and $\Omega \subset \mathbb{R}^{d}, d \in\{1,2,3\}$, is a given domain. The equations are equipped with initial conditions

$$
w(x, 0)=w^{0}(x), \quad x \in \Omega .
$$

The parameter $\varepsilon>0$ is a reference Mach number. In this work, we consider flows at rather low Mach numbers, so $\varepsilon \ll 1$.

It is apparent that for $\varepsilon$ finite but small, this is a singularly perturbed system of equations which renders both the analysis and the numerical treatment of the equations difficult. Expanding $w$ in terms of $\varepsilon$, i.e. assuming a representation of $w$ as

$$
\boldsymbol{w}=\boldsymbol{w}_{(0)}+\varepsilon \boldsymbol{w}_{(1)}+\varepsilon^{2} \boldsymbol{w}_{(2)}+\mathcal{O}\left(\varepsilon^{3}\right)
$$

reveals that under certain conditions, see e.g. [26], the solution $w$ converges to the solution of the incompressible Euler equations with variable density $\boldsymbol{w}_{\text {inc }}:=\left(\rho_{(0)},(\rho \boldsymbol{u})_{(0)}, p_{(2)}\right)^{T}$, viz

$$
\partial_{t}\left(\begin{array}{c}
\rho_{(0)} \\
(\rho \boldsymbol{u})_{(0)} \\
0
\end{array}\right)+\nabla \cdot \underbrace{\left(\begin{array}{c}
(\rho \boldsymbol{u})_{(0)} \\
\rho_{(0)} \boldsymbol{u}_{(0)} \otimes \boldsymbol{u}_{(0)}+p_{(2)} I d \\
\boldsymbol{u}_{(0)}
\end{array}\right)}_{=: g\left(w_{\text {inc }}\right)}=0
$$

with

$$
p_{(0)}:=(\gamma-1) E_{(0)} \equiv \text { const } \quad \text { and } \quad p_{(1)}:=(\gamma-1) E_{(1)} \equiv \text { const. }
$$


Note that also the hydrodynamic pressure $p_{(2)}$ can be represented in terms of the other quantities via

$$
p_{(2)}=(\gamma-1)\left(E_{(2)}-\frac{1}{2} \rho_{(0)}\left\|\boldsymbol{u}_{(0)}\right\|_{2}^{2}\right) .
$$

For more details, we refer to $[25,26,29,32]$. Note that the representation of $w$ as a Hilbert expansion is used throughout the whole paper.

\subsection{Stiff/nonstiff splitting}

As seen before, the Euler equations at low Mach number constitute a system of singularly perturbed differential equations, therefore, they consist of stiff and non-stiff parts. Identifying these correctly can yield very robust and efficient time-stepping procedures. So, assume that $\boldsymbol{f}(\boldsymbol{w})=\widetilde{f}(\boldsymbol{w})+\widehat{f}(\boldsymbol{w})$, with $\widetilde{f}(\boldsymbol{w})$ being the stiff, and $\widehat{f}(\boldsymbol{w})$ being the non-stiff contribution, one can treat the first one implicitly and the second one explicitly in time. For Euler's time integration procedure, this would amount to the IMEX scheme

$$
\frac{\boldsymbol{w}^{n+1}-\boldsymbol{w}^{n}}{\Delta t}+\nabla \cdot\left(\widetilde{f}\left(\boldsymbol{w}^{n+1}\right)+\widehat{f}\left(\boldsymbol{w}^{n}\right)\right)=0 .
$$

In this section, we discuss the extension of the so-called RS-IMEX splitting, previously introduced for the isentropic Euler equations, to the equations at hand. A very important ingredient is a reference solution $(\underline{\text { RS }})$, i.e., a function $w_{\text {ref }}:=\left(\rho_{\text {ref }},(\rho \boldsymbol{u})_{\text {ref }}, E_{\text {ref }}\right)$ such that

$$
\rho-\rho_{\text {ref }}=\mathcal{O}(\varepsilon), \quad \rho \boldsymbol{u}-(\rho \boldsymbol{u})_{\mathrm{ref}}=\mathcal{O}(\varepsilon) \quad \text { and } \quad E-E_{\text {ref }}=\mathcal{O}(\varepsilon) .
$$

Typically, this could be a solution to the limit equations (2.3), or an approximation thereof. This latter approach is pursued in this work, for a discussion on other choices, consult [23]. It is straightforward to apply this splitting also in the context of the full Euler equations:

Definition 2.1 (RS-IMEX splitting). Let a reference solution $w_{\text {ref }}$ be given, then the RSIMEX splitting is given by

$$
\widetilde{f}(w):=f\left(w_{\text {ref }}\right)+\nabla_{w} f\left(w_{\text {ref }}\right)\left(w-w_{\text {ref }}\right) \quad \text { and } \quad \widehat{f}(w):=f(w)-\widetilde{f}(w) .
$$

$\widehat{f}$ and $\widetilde{f}$ are of course well-defined. However, the explicit part does not necessarily give rise to a hyperbolic system as for the isentropic Euler equations:

Lemma 2.1. Consider $d=2$ and $\boldsymbol{n} \in \mathbb{R}^{2}$ with unit length. Then, the eigenvalues of $\nabla_{\boldsymbol{w}} \widehat{\boldsymbol{f}} \cdot \boldsymbol{n}$ are given by

$$
\widehat{\lambda}_{1}=0, \quad \widehat{\lambda}_{2}=\gamma\left(\boldsymbol{u}-\boldsymbol{u}_{r e f}\right) \cdot \boldsymbol{n}, \quad \widehat{\lambda}_{3,4}=\left(2-\frac{\gamma}{2}\right)\left(\boldsymbol{u}-\boldsymbol{u}_{r e f}\right) \cdot \boldsymbol{n} \pm \frac{1}{2} \sqrt{D},
$$


with

$$
D:=(4-4 \gamma)\left\|\boldsymbol{u}-\boldsymbol{u}_{r e f}\right\|^{2}+\gamma^{2}\left(\left(\boldsymbol{u}-\boldsymbol{u}_{r e f}\right) \cdot \boldsymbol{n}\right)^{2} .
$$

For $\gamma>1, D$ can become negative if

$$
|4-4 \gamma|\left\|\boldsymbol{u}-\boldsymbol{u}_{r e f}\right\|^{2}>\gamma^{2}\left(\left(\boldsymbol{u}-\boldsymbol{u}_{r e f}\right) \cdot \boldsymbol{n}\right)^{2} .
$$

Of course this can pose severe problems when applying straightforward solvers designed for hyperbolic problems. In the following, we first discuss some stability-related issues, and then propose a modification of the splitting which avoids complex eigenvalues.

\subsection{Stability considerations}

The explicit flux function of the RS-IMEX scheme is such that it gives rise to a nonhyperbolic system of equations via the complex eigenvalues. In this part, we shortly investigate this influence based on the very simple prototype equation

$$
w_{t}+a w_{x}+i \varepsilon w_{x}=0, \quad(x, t) \in(0,1) \times \mathbb{R}^{+},
$$

with $a \in \mathbb{R}$ and $a \gg \varepsilon \in \mathbb{R}$. We assume that square-integrable initial conditions are given, and that periodic boundary conditions in space are used.

It is our intention to mimic the behavior of an IMEX scheme in the most simple setting. To this end, we assume that the complex convection part, ikw $w_{x}$, is discretized with an explicit method, the real convection part, $a w_{x}$, with an implicit method. Discretization is achieved via a first-order finite volume method using the Rusanov flux with numerical viscosities $\widehat{\alpha}$ and $\widetilde{\alpha}$, respectively. We assume that a time-space grid is given by

$$
\mathcal{T}=\left\{\left(x_{j}, t^{n}\right) \mid x_{j}:=\left(j+\frac{1}{2}\right) \Delta x, j=0, \cdots, N, t^{n}:=n \Delta t, n \in \mathbb{N}\right\}
$$

for constant values $\Delta x\left(=N^{-1}\right)$ and $\Delta t . w_{j}^{n}$ denotes an approximation to $w\left(x_{j}, t^{n}\right)$. Ultimately, this yields the method

$$
w_{j}^{n+1}=w_{j}^{n}-\frac{a \Delta t}{2 \Delta x}\left(w_{j+1}^{n+1}-w_{j-1}^{n+1}\right)+\frac{\widetilde{\alpha} \Delta t}{2 \Delta x}\left(w_{j+1}^{n+1}+w_{i-1}^{n+1}-2 w_{j}^{n}\right)-\frac{i \varepsilon \Delta t}{2 \Delta x}\left(w_{j+1}^{n}-w_{j-1}^{n}\right) .
$$

Note that we have set $\widehat{\alpha}$ to zero for a clearer exposition; qualitatively, this does not influence the results.

The discrete initial conditions can be written as

$$
w_{j}^{0}=\sum_{k=-\lfloor N / 2\rfloor}^{\lfloor N / 2\rfloor} c_{k} e^{2 \pi i k x_{j}}
$$


for some given discrete Fourier coefficients $c_{k}$. Performing a von-Neumann analysis reveals that the method would be stable if

$$
\frac{\left|1+\frac{\varepsilon \Delta t}{\Delta x} \sin (2 \pi k \Delta x)\right|}{\left|1+\frac{i a \Delta t}{\Delta x} \sin (2 \pi k \Delta x)+\frac{\tilde{\alpha} \Delta t}{\Delta x}(1-\cos (2 \pi k \Delta x))\right|} \leq 1, \quad \forall-N / 2 \leq k \leq N / 2 .
$$

There does not exist a finite CFL number such that this is fulfilled for all meshes. However, for a given and fixed grid, one can, at least in theory, always choose $\varepsilon$ in such a way that this requirement is fulfilled. Still, computing is always done with a fixed $\varepsilon$ on a fixed grid, and so there is certainly need for a modification of the splitting, which will be presented in the next section.

\subsection{Modified RS-IMEX splitting}

Following the ideas of Bispen et al., see [5], we modify the explicit part of the RS-IMEX splitting in such a way that the eigenvalues of the non-stiff part remain real. To this end, we drop the explicit contribution of pressure. Thereby, we introduce a small (in terms of ع) modeling error.

Remark 2.1. Written out explicitly, the $\widehat{f}(w)$ given in Definition 2.1 is given as

$$
\widehat{\boldsymbol{f}}(\boldsymbol{w}):=\left(\begin{array}{c}
0 \\
\rho\left(\boldsymbol{u}-\boldsymbol{u}_{\mathrm{ref}}\right) \otimes\left(\boldsymbol{u}-\boldsymbol{u}_{\mathrm{ref}}\right)+\frac{1}{\varepsilon^{2}} \widehat{p} I d \\
\left(\boldsymbol{u}-\boldsymbol{u}_{\mathrm{ref}}\right) \frac{\left(\rho_{\mathrm{ref}} E-E_{\mathrm{ref}} \rho\right)}{\rho_{\mathrm{ref}}}+\widehat{\boldsymbol{u} p}
\end{array}\right),
$$

with

$$
\widehat{p}:=-(\gamma-1) \frac{\varepsilon^{2}}{2} \rho\left\|\boldsymbol{u}_{\mathrm{ref}}-\boldsymbol{u}\right\|^{2}
$$

and

$$
\begin{aligned}
\widehat{\boldsymbol{u p}}:= & (\gamma-1)\left(\boldsymbol{u}-\boldsymbol{u}_{\text {ref }}\right)\left(E-\frac{\rho}{\rho_{\text {ref }}} E_{\text {ref }}\right) \\
& +(\gamma-1) \frac{\varepsilon^{2}}{2} \rho\left[\left(\left\|\boldsymbol{u}_{\text {ref }}\right\|_{2}^{2}-\|\boldsymbol{u}\|_{2}^{2}\right)\left(\boldsymbol{u}-\boldsymbol{u}_{\text {ref }}\right)-\left\|\boldsymbol{u}-\boldsymbol{u}_{\text {ref }}\right\|_{2}^{2} \boldsymbol{u}_{\text {ref }}\right] .
\end{aligned}
$$

For $w$ being the exact solution, there holds

$$
\widehat{p}=\mathcal{O}\left(\varepsilon^{4}\right) \quad \text { and } \quad \widehat{u p}=\mathcal{O}\left(\varepsilon^{2}\right)+\mathcal{O}\left(\varepsilon^{4}\right) .
$$

Thus, dropping the $\mathcal{O}\left(\varepsilon^{4}\right)$ terms should lead to an error in $\mathcal{O}\left(\varepsilon^{2}\right)$, since $\hat{p}$ is multiplied with $\varepsilon^{-2}$. This will yield a hyperbolic non-stiff contribution, at the price of a modeling error. 
Definition 2.2 (RS-IMEX splitting (modified)). Let a reference solution $w_{\text {ref }}$ be given, then the modified RS-IMEX splitting for the Euler equations is defined by

$$
\begin{aligned}
& \widetilde{f}(\boldsymbol{w}):=f\left(\boldsymbol{w}_{\mathrm{ref}}\right)+\nabla_{\boldsymbol{w}} f\left(\boldsymbol{w}_{\mathrm{ref}}\right)\left(\boldsymbol{w}-\boldsymbol{w}_{\mathrm{ref}}\right) \\
& \text { and } \quad \widehat{f}_{\text {mod }}(\boldsymbol{w}):=\left(\begin{array}{c}
0 \\
\rho\left(\boldsymbol{u}-\boldsymbol{u}_{\mathrm{ref}}\right) \otimes\left(\boldsymbol{u}-\boldsymbol{u}_{\mathrm{ref}}\right) \\
\gamma\left(\boldsymbol{u}-\boldsymbol{u}_{\mathrm{ref}}\right) \frac{\left(\rho_{\mathrm{ref}} E-E_{\mathrm{ref}} \rho\right)}{\rho_{\mathrm{ref}}}
\end{array}\right)
\end{aligned}
$$

Lemma 2.2. Consider $d=2$ and $\boldsymbol{n} \in \mathbb{R}^{2}$ with unit length. Then, the eigenvalues of $\nabla_{\boldsymbol{w}} \widehat{f}_{\mathrm{mod}} \cdot \boldsymbol{n}$ are given by

$$
\widehat{\lambda}_{1}=0, \quad \widehat{\lambda}_{2}=2\left(\boldsymbol{u}-\boldsymbol{u}_{r e f}\right) \cdot \boldsymbol{n}, \quad \widehat{\lambda}_{3}=\left(\boldsymbol{u}-\boldsymbol{u}_{r e f}\right) \cdot \boldsymbol{n} \quad \text { and } \quad \widehat{\lambda}_{4}=\gamma\left(\boldsymbol{u}-\boldsymbol{u}_{r e f}\right) \cdot \boldsymbol{n},
$$

and are real.

Remark 2.2. The eigenvalues of the explicit part are similar to the eigenvalues of the explicit part of the RS-IMEX splitting applied to the isentropic Euler equations, see e.g. [22].

Remark 2.3. For the sake of completeness, we also give the explicit expression for the flux $\widetilde{f}(w)$ :

$$
\begin{aligned}
\widetilde{f}(\boldsymbol{w}):= & \left(\begin{array}{c}
\rho \boldsymbol{u} \\
\rho\left(\boldsymbol{u}_{\mathrm{ref}} \otimes \boldsymbol{u}+\boldsymbol{u} \otimes \boldsymbol{u}_{\mathrm{ref}}-\boldsymbol{u}_{\mathrm{ref}} \otimes \boldsymbol{u}_{\mathrm{ref}}\right) \\
\gamma\left(\frac{\rho}{\rho_{\mathrm{ref}}}\left(\boldsymbol{u}-\boldsymbol{u}_{\mathrm{ref}}\right) E_{\mathrm{ref}}+\boldsymbol{u}_{\mathrm{ref}} E\right)
\end{array}\right) \\
& +(\gamma-1)\left(\begin{array}{c}
0 \\
\frac{1}{\varepsilon^{2}}\left[E-\frac{\varepsilon^{2}}{2} \rho\left(\|\boldsymbol{u}\|_{2}^{2}-\left\|\boldsymbol{u}-\boldsymbol{u}_{\mathrm{ref}}\right\|_{2}^{2}\right)\right] I d \\
-\frac{\varepsilon^{2}}{2} \rho\left[\left(\|\boldsymbol{u}\|_{2}^{2}-\left\|\boldsymbol{u}-\boldsymbol{u}_{\mathrm{ref}}\right\|_{2}^{2}\right) \boldsymbol{u}_{\mathrm{ref}}+\left\|\boldsymbol{u}_{\mathrm{ref}}\right\|_{2}^{2}\left(\boldsymbol{u}-\boldsymbol{u}_{\mathrm{ref}}\right)\right]
\end{array}\right) .
\end{aligned}
$$

Note that the implicit part is linear in $w$. The associated eigenvalues are those of the original system, evaluated at reference state.

From now on, we will drop the subscript 'mod' in the modified RS-IMEX flux; the context will be clear.

\section{Numerical discretization}

\subsection{Semi discrete scheme}

We have already in (2.5) shortly touched upon the issue of time integration schemes. The focus is of course on higher order, and therefore, IMEX Euler is not sufficient. In this work, we use globally stiffly accurate IMEX Runge-Kutta methods of type CK with uniform internal timestep vector, see e.g. $[2,7,24,28]$. Based on the split PDE

$$
\partial_{t} \boldsymbol{w}+\nabla \cdot[\widetilde{f}(\boldsymbol{w})+\widehat{f}(w)]=0,
$$


with splitting as in Definition 2.2 (modified RS-IMEX), we can formulate the schemes as follows:

Definition 3.1 (IMEX Runge-Kutta method). Let a globally stiffly accurate IMEX RungeKutta method be given by its Butcher tableau. Let the implicit matrix $\widetilde{A}$ be a lower triangular matrix with $\widetilde{A}_{i i} \neq 0$ for $i>1$, and $s$ denote the number of stages. Then, for every $n=0, \cdots$, do the following:

1. For $i=1, \cdots, s$ solve at time instance $t^{n, j}:=t^{n}+c^{j} \Delta t^{n}$

$$
\boldsymbol{w}^{n, i}=\boldsymbol{w}^{n}-\Delta t^{n} \sum_{j=1}^{i} \widetilde{A}_{i, j} \nabla \cdot \widetilde{\boldsymbol{f}}\left(\boldsymbol{w}^{n, j}\right)-\Delta t^{n} \sum_{j=1}^{i-1} \widehat{A}_{i, j} \nabla \cdot \widehat{\boldsymbol{f}}\left(\boldsymbol{w}^{n, j}\right) .
$$

2. Set $\boldsymbol{w}^{n+1}:=\boldsymbol{w}^{n, s}$.

Remark 3.1. For the numerical results presented in this work, we use the following schemes:

- IMEX-ARS-222, a second order scheme given in [2];

- IMEX-ARS-443, a third order scheme given in [2];

- IMEX-ARK-4A2, a fourth order scheme given in [28].

Note that in all the numerical results, we work with adaptive time steps $\Delta t^{n}$ dictated by the convective CFL condition, see (3.18).

\subsection{Asymptotic consistency of semi discrete scheme}

A very important property with respect to singularly perturbed equations is asymptotic consistency. This property guarantees that the $\varepsilon \rightarrow 0$ limit of the discretization is a consistent discretization of the incompressible Euler equations (2.3). For more details and other applications, we refer to $[20,21,29]$ and the references therein. In this section, we prove that the semi-discrete (in time) method from Definition 3.1 is asymptotically consistent if the modified RS-IMEX splitting is used. The proof is done in two steps:

- First, it is shown that a fully implicit semi-discretization is asymptotically consistent. A fully implicit discretization amounts to taking $\widetilde{f}(\boldsymbol{w})=f(\boldsymbol{w})$, and $\widehat{f}(\boldsymbol{w})=0$ in Definition 3.1.

- Second, it is shown that the limiting equation of the modified RS-IMEX approach and the fully implicit method have the same solution. This automatically renders the method asymptotically preserving. 


\subsubsection{Fully implicit discretization}

Applying an implicit method to the Euler equations yields the definition of the stages

$$
\widetilde{\boldsymbol{w}}^{n, i}=\widetilde{\boldsymbol{w}}^{n}-\Delta t^{n} \sum_{j=1}^{i} \widetilde{A}_{i, j} \nabla \cdot \boldsymbol{f}\left(\widetilde{\boldsymbol{w}}^{n, j}\right),
$$

where $\widetilde{\boldsymbol{w}}:=(\widetilde{\rho}, \widetilde{\rho \boldsymbol{u}}, \widetilde{E})^{T}$ and $f$ is given by Eq. (2.1). Note that we use the identifier $\widetilde{(\cdot)}$ to differentiate between the numerical solution obtained by a fully implicit discretization and the numerical solution obtained by the RS-IMEX splitting.

Lemma 3.1. Assume that $\widetilde{\boldsymbol{w}}^{n, j}$ is well-prepared for $j<i$, i.e., there holds

$$
\widetilde{E}^{n, j}=\mathrm{const}+\mathcal{O}\left(\varepsilon^{2}\right) \quad \text { and } \quad \nabla \cdot \widetilde{\boldsymbol{u}}^{n, j}=\mathcal{O}(\varepsilon) .
$$

Furthermore, let the boundary conditions be such that

$$
\int_{\partial \Omega} \widetilde{\boldsymbol{u}}^{n, i} \cdot \boldsymbol{n} \mathrm{d} \sigma=0
$$

If $\widetilde{\boldsymbol{w}}^{n, i}$ has a Hilbert expansion, then also $\widetilde{\boldsymbol{w}}^{n, i}$ is well-prepared. Furthermore, the $\varepsilon \rightarrow 0$ limit of scheme (3.1) is a consistent discretization of the incompressible Euler equations.

Proof. We assume that all quantities are given as an asymptotic expansion, i.e.

$$
\begin{aligned}
& \widetilde{\rho}^{n, i}=\widetilde{\rho}_{(0)}^{n, i}+\varepsilon \widetilde{\rho}_{(1)}^{n, i}+\varepsilon^{2} \widetilde{\rho}_{(2)}^{n, i}+\mathcal{O}\left(\varepsilon^{3}\right), \\
& \widetilde{\boldsymbol{u}}^{n, i}=\widetilde{\boldsymbol{u}}_{(0)}^{n, i}+\varepsilon \widetilde{\boldsymbol{u}}_{(1)}^{n, i}+\varepsilon^{2} \widetilde{\boldsymbol{u}}_{(2)}^{n, i}+\mathcal{O}\left(\varepsilon^{3}\right), \\
& \widetilde{E}^{n, i}=\widetilde{E}_{(0)}^{n, i}+\varepsilon \widetilde{E}_{(1)}^{n, i}+\varepsilon^{2} \widetilde{E}_{(2)}^{n, i}+\mathcal{O}\left(\varepsilon^{3}\right),
\end{aligned}
$$

insert this in the numerical method given in Eq. (3.1) and vary in terms of $\varepsilon$. By this we obtain

$$
\begin{aligned}
& \widetilde{\rho}_{(0)}^{n, i}=\widetilde{\rho}_{(0)}^{n}-\Delta t^{n} \sum_{j=1}^{i} \widetilde{A}_{i, j} \nabla \cdot \widetilde{\rho \boldsymbol{u}}_{(0)^{\prime}}^{n, j} \\
& \widetilde{\rho \boldsymbol{u}}_{(0)}^{n, i}=\widetilde{\rho \boldsymbol{u}}_{(0)}^{n}-\Delta t^{n} \sum_{j=1}^{i} \widetilde{A}_{i, j} \nabla \cdot\left[\widetilde{\rho}_{(0)}^{n, j} \widetilde{\boldsymbol{u}}_{(0)}^{n, j} \otimes \widetilde{\boldsymbol{u}}_{(0)}^{n, j}+(\gamma-1)\left(\widetilde{E}_{(2)}^{n, j}-\frac{1}{2} \widetilde{\rho}_{(0)}^{n, j}\left\|\widetilde{\boldsymbol{u}}_{(0)}^{n, j}\right\|_{2}^{2}\right) I d\right], \\
& \widetilde{E}_{(0)}^{n, i}=\widetilde{E}_{(0)}^{n}-\Delta t^{n} \sum_{j=1}^{i} \widetilde{A}_{i, j} \nabla \cdot \gamma \widetilde{\boldsymbol{u}}_{(0)}^{n, j} \widetilde{E}_{(0)^{\prime}}^{n, j} \\
& 0=\Delta t^{n} \sum_{j=1}^{i} \widetilde{A}_{i, j} \nabla \cdot(\gamma-1) \widetilde{E}_{(0)}^{n, j} I d \\
& 0=\Delta t^{n} \sum_{j=1}^{i} \widetilde{A}_{i, j} \nabla \cdot(\gamma-1) \widetilde{E}_{(1)}^{n, j} I d .
\end{aligned}
$$


All previous stages are assumed to be well prepared, i.e. they fulfill

$$
E_{(0)}^{n, j} \equiv E_{(0)}, \quad E_{(1)}^{n, j} \equiv E_{(1)} \quad \text { and } \quad \nabla \cdot \widetilde{\boldsymbol{u}}_{(0)}^{n, j}=0, \quad j<i .
$$

Then, Eqs. (3.6) and (3.7) directly reduce to

$$
0=\widetilde{A}_{i, i} \nabla \widetilde{E}_{(0)}^{n, i} \quad \text { and } \quad 0=\widetilde{A}_{i, i} \nabla \widetilde{E}_{(1)}^{n, i} .
$$

Thus, $\widetilde{E}_{(0)}^{n, i}$ and $\widetilde{E}_{(1)}^{n, i}$ are constant in space for all $i$. (Note that if $A_{11}=0$, then this is implied due to the prerequisites.) Next, we consider Eq. (3.5). Due to the previous results and well prepared previous stages we obtain

$$
\widetilde{E}_{(0)}^{n, i}-\widetilde{E}_{(0)}^{n}=-\Delta t^{n} \widetilde{A}_{i, i} \widetilde{E}_{(0)}^{n, j} \gamma \nabla \cdot \widetilde{\boldsymbol{u}}_{(0)}^{n, i} \cdot
$$

Integrating over the whole domain, using Gauss theorem and well-prepared boundary conditions, see Lemma 3.1, we can conclude that

$$
\widetilde{E}_{(0)}^{n, i}=\widetilde{E}_{(0)}^{n} \equiv \widetilde{E}_{(0)}
$$

Considering again Eq. (3.5), there follows

$$
\nabla \cdot \widetilde{\boldsymbol{u}}_{(0)}^{n, i}=0
$$

Thus, $\widetilde{\boldsymbol{w}}^{n, i}$ fulfills all conditions of well-prepared initial data, see Eq. (3.2). It is straightforward to see that the remaining equations

$$
\begin{aligned}
& \widetilde{\rho}_{(0)}^{n, i}=\widetilde{\rho}_{(0)}^{n}-\Delta t^{n} \sum_{j=1}^{i} \widetilde{A}_{i, j} \nabla \cdot \widetilde{\rho \boldsymbol{u}}_{(0)}^{n, j}, \\
& \widetilde{\rho \boldsymbol{u}}_{(0)}^{n, i}=\widetilde{\rho \boldsymbol{u}}_{(0)}^{n}-\Delta t^{n} \sum_{j=1}^{i} \widetilde{A}_{i, j} \nabla \cdot\left[\widetilde{\rho}_{(0)}^{n, j} \widetilde{\boldsymbol{u}}_{(0)}^{n, j} \otimes \widetilde{\boldsymbol{u}}_{(0)}^{n, j}+(\gamma-1)\left(\widetilde{E}_{(2)}^{n, j}-\frac{1}{2} \widetilde{\rho}_{(0)}^{n, j}\left\|\widetilde{\boldsymbol{u}}_{(0)}^{n, j}\right\|_{2}^{2}\right) I d\right]
\end{aligned}
$$

constitute a consistent discretization of the incompressible Euler equations, see Eq. (2.3), with $p_{(2)}$ given by Eq. (2.4). Therefore, the method is asymptotically consistent.

\subsubsection{Modified RS-IMEX splitting}

Based on the previously shown result, we show that the overall method is asymptotically consistent. First, the limiting method is derived. 
Lemma 3.2. The formal $\varepsilon \rightarrow 0$ limit of a globally stiffly accurate IMEX Runge-Kutta scheme, see Definition 3.1, coupled with the modified RS-IMEX splitting is given by

$$
\begin{aligned}
\rho_{(0)}^{n, i}= & \rho_{(0)}^{n}-\Delta t^{n} \sum_{j=1}^{i} \widetilde{A}_{i, j} \nabla \cdot \rho \boldsymbol{u}_{(0)^{\prime}}^{n, j} \\
\rho \boldsymbol{u}_{(0)}^{n, i}= & \rho \boldsymbol{u}_{(0)}^{n}-\Delta t^{n} \sum_{j=1}^{i-1} \widehat{A}_{i, j} \nabla \cdot\left[\rho_{(0)}^{n, j}\left(\boldsymbol{u}_{(0)}^{n, j}-\boldsymbol{u}_{r e f}\right) \otimes\left(\boldsymbol{u}_{(0)}^{n, j}-\boldsymbol{u}_{r e f}\right)\right] \\
& -\Delta t^{n} \sum_{j=1}^{i} \widetilde{A}_{i, j} \nabla \cdot\left[\rho_{(0)}^{n, j}\left(\boldsymbol{u}_{r e f} \otimes \boldsymbol{u}_{(0)}^{n, j}+\boldsymbol{u}_{(0)}^{n, j} \otimes \boldsymbol{u}_{r e f}-\boldsymbol{u}_{r e f} \otimes \boldsymbol{u}_{r e f}\right)\right] \\
& -\Delta t^{n} \sum_{j=1}^{i} \widetilde{A}_{i, j} \nabla \cdot(\gamma-1)\left[E_{(2)}^{n, j}-\frac{\rho_{(0)}^{n, j}}{2}\left(\left\|\boldsymbol{u}_{(0)}^{n, j}\right\|_{2}^{2}-\left\|\boldsymbol{u}_{(0)}^{n, j}-\boldsymbol{u}_{r e f}\right\|_{2}^{2}\right)\right] I d, \\
E_{(0)}^{n, i}= & E_{(0)}^{n}-\Delta t^{n} \sum_{j=1}^{i-1} \widehat{A}_{i, j} \nabla \cdot \gamma\left(\boldsymbol{u}_{(0)}^{n, j}-\boldsymbol{u}_{r e f} \frac{\rho_{r e f} E_{(0)}^{n, j}-E_{r e f} \rho_{(0)}^{n, j}}{\rho_{r e f}}\right. \\
& -\Delta t^{n} \sum_{j=1}^{i} \widetilde{A}_{i, j} \nabla \cdot \gamma\left(\frac{\rho_{(0)}^{n, j}}{\rho_{r e f}}\left(\boldsymbol{u}_{(0)}^{n, j}-\boldsymbol{u}_{r e f}\right) E_{r e f}+\boldsymbol{u}_{r e f} E_{(0)}^{n, j}\right) \\
0= & \Delta t^{n} \sum_{j=1}^{i} \widetilde{A}_{i, j} \nabla \cdot(\gamma-1) E_{(0)}^{n, j} I d, \\
0= & \Delta t^{n} \sum_{j=1}^{i} \widetilde{A}_{i, j} \nabla \cdot(\gamma-1) E_{(1)}^{n, j} I d .
\end{aligned}
$$

Also $\rho_{\text {ref }}, \boldsymbol{u}_{\text {ref }}$ and $E_{\text {ref }}$ are time-dependent; for the ease of presentation we have neglected the superscripts $n, i$. It is to be understood that they are evaluated at time instances $t^{n, j}=t^{n}+\Delta t^{n} c^{j}$, where $c^{j}$ is the partial time step dictated by the Runge-Kutta method, see Definition 3.1.

Proof. The lemma is a straightforward computation, assuming the Hilbert expansions

$$
\begin{aligned}
& \rho^{n, i}=\rho_{(0)}^{n, i}+\varepsilon \rho_{(1)}^{n, i}+\varepsilon^{2} \rho_{(2)}^{n, i}+\mathcal{O}\left(\varepsilon^{2}\right), \\
& \boldsymbol{u}^{n, i}=\boldsymbol{u}_{(0)}^{n, i}+\varepsilon \boldsymbol{u}_{(1)}^{n, i}+\varepsilon^{2} \boldsymbol{u}_{(2)}^{n, i}+\mathcal{O}\left(\varepsilon^{2}\right), \\
& E^{n, i}=E_{(0)}^{n, i}+\varepsilon E_{(1)}^{n, i}+\varepsilon^{2} E_{(2)}^{n, i}+\mathcal{O}\left(\varepsilon^{2}\right)
\end{aligned}
$$

for every $i$, and collecting terms in $\varepsilon$.

Remark 3.2. It will turn out that we can neglect difference terms of reference solution and discrete solution. Note that

$$
\boldsymbol{u}_{\mathrm{ref}} \otimes \boldsymbol{u}_{(0)}^{n, j}+\boldsymbol{u}_{(0)}^{n, j} \otimes \boldsymbol{u}_{\mathrm{ref}}-\boldsymbol{u}_{\mathrm{ref}} \otimes \boldsymbol{u}_{\mathrm{ref}}=\boldsymbol{u}_{(0)}^{n, j} \otimes \boldsymbol{u}_{\mathrm{ref}}+\boldsymbol{u}_{\mathrm{ref}} \otimes\left(\boldsymbol{u}_{(0)}^{n, j}-\boldsymbol{u}_{\mathrm{ref}}\right) .
$$


In this sense, Eqs. (3.8)-(3.10) can be simplified to give

$$
\begin{aligned}
\rho_{(0)}^{n, i}= & \rho_{(0)}^{n}-\Delta t^{n} \sum_{j=1}^{i} \widetilde{A}_{i, j} \nabla \cdot \rho \boldsymbol{u}_{(0)^{\prime}}^{n, j} \\
\rho \boldsymbol{u}_{(0)}^{n, i}= & \rho \boldsymbol{u}_{(0)}^{n}-\Delta t^{n} \sum_{j=1}^{i} \widetilde{A}_{i, j} \nabla \cdot\left[\rho_{(0)}^{n, j} \boldsymbol{u}_{(0)}^{n, j} \otimes \boldsymbol{u}_{\mathrm{ref}}+(\gamma-1)\left(E_{(2)}^{n, j}-\frac{\rho_{(0)}^{n, j}}{2}\left\|\boldsymbol{u}_{(0)}^{n, j}\right\|_{2}^{2}\right) I d\right] \\
& +\mathcal{O}\left(\boldsymbol{u}_{(0)}^{n, j}-\boldsymbol{u}_{\mathrm{ref}}\right), \\
E_{(0)}^{n, i}= & E_{(0)}^{n}-\Delta t^{n} \sum_{j=1}^{i} \widetilde{A}_{i, j} \nabla \cdot \gamma \boldsymbol{u}_{\mathrm{ref}} E_{(0)}^{n, j}+\mathcal{O}\left(\boldsymbol{u}_{(0)}^{n, j}-\boldsymbol{u}_{\mathrm{ref}}\right) .
\end{aligned}
$$

Note the apparent similarity with the implicit method, see Lemma 3.1.

With this, we can now, under a restriction on the reference solution, show that the overall method is asymptotically consistent. For this, we assume that the reference solution is computed with the fully implicit method given in Lemma 3.1 and then show that the method given in Lemma 3.2 is solved by the fully implicit solution.

Lemma 3.3. Assume the conditions of Lemma 3.1. Assume furthermore that the reference solution is computed by the fully implicit method given in Lemma 3.1, i.e.

$$
\rho_{\text {ref }}\left(t^{n, i}\right):=\widetilde{\rho}_{(0)^{\prime}}^{n, i} \quad(\rho \boldsymbol{u})_{\text {ref }}\left(t^{n, i}\right):={\widetilde{(\rho \boldsymbol{u})_{(0)}^{n, i}}}_{(0)}^{n+} \quad \text { and } \quad E_{r e f}\left(t^{n, i}\right):=\widetilde{E}_{(0)}^{n, i} \equiv E_{(0)} .
$$

Then, the solution obtained by the fully implicit method, see again Lemma 3.1, is a solution of the limiting equations obtained by the modified RS-IMEX splitting, see Lemma 3.2, and in particular Remark 3.2.

Proof. Plugging the limit solution $\boldsymbol{w}_{\text {ref }}\left(t^{n, i}\right):=\widetilde{\boldsymbol{w}}_{(0)}^{n, i}$ into (3.13)-(3.15) directly yields the claim.

Overall, we can conclude that a globally stiffly accurate IMEX Runge-Kutta scheme coupled with the modified RS-IMEX splitting is asymptotically consistent if the corresponding implicit discretization is asymptotically consistent, which is indeed the case.

Corollary 3.1. A globally stiffly accurate IMEX Runge-Kutta scheme coupled with the modified RS-IMEX splitting is asymptotically consistent.

\subsection{Fully discrete scheme}

In general we use the same discretization as previously used in [23] and [37] for the isentropic Euler equations. This means that we couple a high order IMEX Runge-Kutta 
scheme with a discontinuous Galerkin method. Details about the used nodal discontinuous Galerkin spectral element method can be found in [17,27]. For problems with strong gradients, we use a Finite Volume method described in [33] with $2^{\text {nd }}$ order in space reconstruction and MinMod slope limiting [31]. For the fluxes across the boundaries of the DG elements and the numerical fluxes of the finite volume method we use a Lax-Friedrichs type Riemann solver described by

$$
\widetilde{f}^{*}=\frac{1}{2}\left(\widetilde{f}_{n}\left(w^{+}\right)+\widetilde{f}_{n}\left(w^{-}\right)+\widetilde{\Lambda}\left(w^{+}-w^{-}\right)\right), \quad \widetilde{\Lambda}:=\operatorname{Diag}\left\{\frac{1}{\varepsilon}, 1,1,1, \frac{1}{\varepsilon^{2}}\right\},
$$

for the implicit part and

$$
\widehat{f}^{*}=\frac{1}{2}\left(\widehat{f}_{n}\left(\boldsymbol{w}^{+}\right)+\widehat{f}_{n}\left(\boldsymbol{w}^{-}\right)+\widehat{\Lambda}\left(\boldsymbol{w}^{+}-\boldsymbol{w}^{-}\right)\right), \quad \widehat{\Lambda}:=2 \varepsilon \operatorname{Diag}\{1, \cdots, 1\},
$$

for the explicit part with $(\cdot)_{n}$ denoting the flux in face normal direction and $(\cdot)^{+}$and $(\cdot)^{-}$ denoting the left and right state at a cell interface.

As described in Section 2 the RS-IMEX splitting requires the solution of the incompressible Euler equations given in Eq. (2.3). For the incompressible solver we adopt the method for non-variable density incompressible flow which has been used in [37]. Here, the Riemann solver has to be changed to a Lax-Friedrichs type Riemann solver with

$$
g^{*}=\frac{1}{2}\left(g_{n}\left(w_{\text {inc }}{ }^{+}\right)+g_{n}\left(w_{\text {inc }}{ }^{-}\right)+\Lambda_{\text {inc }}\left(w_{\text {inc }}{ }^{+}-w_{\text {inc }}{ }^{-}\right)\right), \quad \Lambda_{\text {inc }}=\operatorname{Diag}\left\{1, \cdots, 1, \frac{1}{\gamma}\right\} .
$$

For the solution of the equation system arising from the IMEX Runge-Kutta method we use a Jacobian-free Newton-GMRES method with analytical block-Jacobi preconditioner which is described in more detail in [37]. Note that the time step is chosen according to a CFL number with respect to the velocity $\boldsymbol{u}^{n}$

$$
\Delta t^{n}=\mathrm{CFL} \frac{\Delta x}{(2 \mathcal{N}+1) \max \left|\boldsymbol{u}^{n}\right|},
$$

with the polynomial degree of the solution approximation $\mathcal{N}$ being zero for the finite volume method.

\section{Modeling considerations}

The original RS-IMEX splitting, see Definition 2.1, is such that $f(\boldsymbol{w})=\widehat{f}(\boldsymbol{w})+\widetilde{f}(\boldsymbol{w})$, so there is no modeling error introduced. However, the modified RS-IMEX splitting, see Definition 2.2, is such that the stiff and non-stiff contributions do not add up to the full Euler flux function. Hence, this gives in principal rise to an inconsistent scheme; for a low-Mach solution, the inconsistency scales with $\mathcal{O}\left(\varepsilon^{2}\right)$, it can therefore also be seen as a modeling error. In this chapter, we investigate the influence of this modification. 


\subsection{2d low-Mach vortex}

In this first example, we consider an exact solution. We choose $\gamma=2$, background velocity $u_{\infty}=(1,1)$ and background energy $E_{\infty}=1$. The initial conditions

$$
\begin{aligned}
& \rho^{0}(x)=\left(1-\frac{\gamma-1}{8 \gamma \pi^{2}} \varepsilon^{2} e^{1-\|x\|_{2}^{2}}\right)^{\frac{1}{\gamma-1}}, \quad \boldsymbol{u}^{0}(x)=u_{\infty}+\varepsilon\left(\begin{array}{c}
-x_{2} \\
x_{1}
\end{array}\right) \frac{e^{\frac{1}{2}\left(1-\|x\|_{2}^{2}\right)}}{2 \pi}, \\
& E^{0}(x)=E_{\infty}+\varepsilon^{2}\left(\frac{\left(\rho^{0}\right)^{\gamma}}{\gamma-1}+\frac{1}{2} \rho^{0}\left\|\boldsymbol{u}^{0}\right\|_{2}^{2}\right),
\end{aligned}
$$

lead to the exact solution of the Euler equations (2.1) via

$$
\rho(x, t)=\rho^{0}\left(x-u_{\infty} t\right), \quad \boldsymbol{u}(\boldsymbol{x}, t)=\boldsymbol{u}^{0}\left(\boldsymbol{x}-\boldsymbol{u}_{\infty} t\right) \quad \text { and } \quad E(\boldsymbol{x}, t)=E^{0}\left(\boldsymbol{x}-\boldsymbol{u}_{\infty} t\right) .
$$

For these particular values, the incompressible solution is the constant state

$$
\rho_{(0)}=1, \quad \boldsymbol{u}_{(0)}=\boldsymbol{u}_{\infty}, \quad p_{(2)}=0 .
$$

To avoid dominating errors from the boundary conditions, we choose a sufficiently large domain $\Omega=[-10,10]^{2}$; and periodic boundary conditions. Note that the solution at the boundary is close to a constant for the initial solution.

This testcase allows us to

- validate the method concerning its order of convergence;

- verify the asymptotic preserving property of the scheme;

- illustrate the effect of complex eigenvalues;

- investigate the influence of the modification described by Definition 2.2 and hence the inconsistency introduced into the scheme.

Fig. 1 illustrates the spatial order of convergence for the DG scheme at different Mach numbers. We see that in general the expected $3^{r d}$ and $4^{\text {th }}$ order are obtained and only slight differences between the scheme with and without modification are present. Note that for small errors and small Mach numbers, the correct order is not reached as round off errors caused by machine accuracy are dominating. Another observation can be made for $\varepsilon=1$ and $\varepsilon=10^{-1}$, where differences between the two schemes are visible. Fig. 1 shows that starting from a certain spatial resolution, the error does not decrease any more for the scheme with modification, whereas the unmodified scheme still shows the correct order of convergence. From this we can see that here the modeling error introduced by the modification is dominating the discretization error. We can conclude that this modeling error only affects the solution for relatively large Mach numbers and fine discretization parameters, as can be expected from the scaling of the modeling error. In order to highlight this, Fig. 2 (left) shows the scaling of the errors of the conservative variables with 

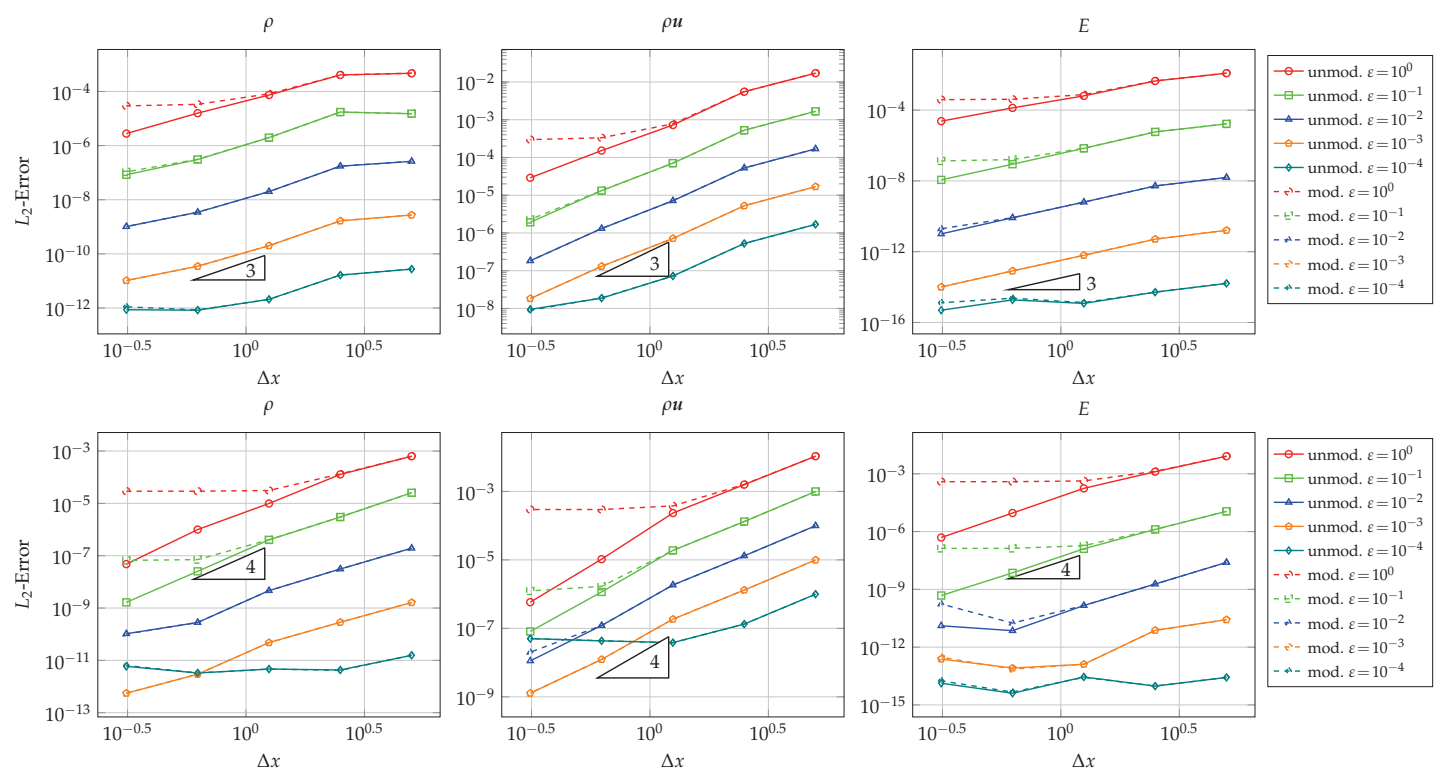

Figure 1: $h$-convergence of conservative variables for DG RS-IMEX with and without modification evaluated with low Mach vortex (top: $\mathcal{N}=2$, IMEX-ARS-443 [2], CFL $=0.5$; bottom: $\mathcal{N}=3$, IMEX-ARK-4A2 [28], $\mathrm{CFL}=0.5)$. Errors are calculated at $t_{\text {end }}=0.1$.
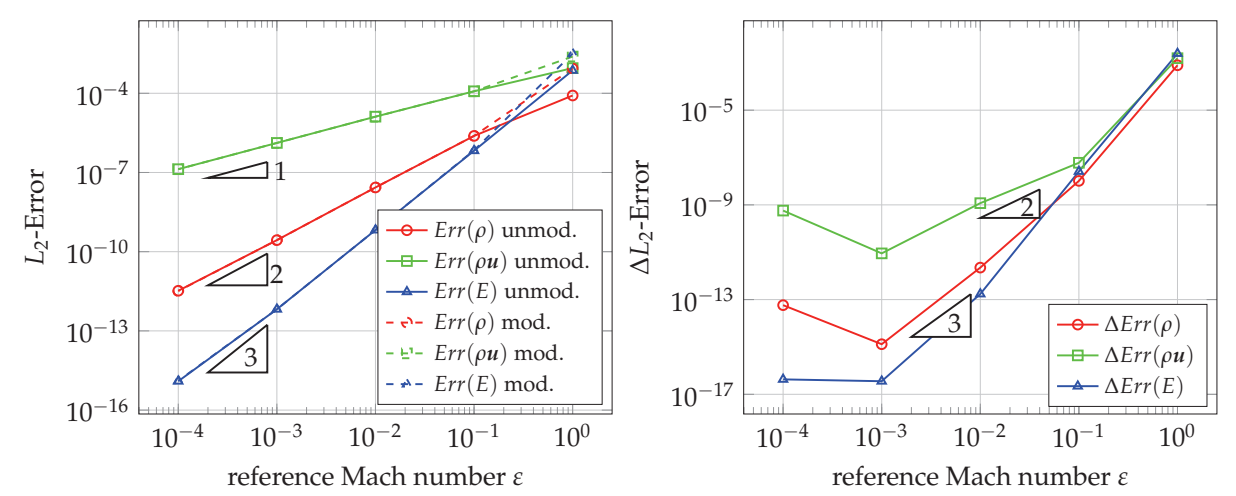

Figure 2: Scaling of Errors with respect to $\varepsilon$ for low Mach vortex using DG RS-IMEX with and without modification (left). Difference between the two schemes (right). Errors are obtained on grid with $16^{2}$ elements, $\mathcal{N}=2$, IMEX-ARS-443, CFL $=0.5$ and $t_{\text {end }}=1$.

respect to the Mach number. We see that the scaling is as described by Eq. (4.1): The error scales with $\varepsilon^{2}$ in density, $\varepsilon^{1}$ in momentum and $\varepsilon^{3}$ in energy. This behavior numerically verifies the asymptotic consistency of the scheme. On the right of Fig. 2, the difference of the scheme with and without modification is visualized. It shows that for Mach numbers $\varepsilon \geq 10^{-3}$, the difference between the schemes scales with $\varepsilon^{2}$ or better. This is expected as the terms deleted in the modified RS-IMEX are in $\mathcal{O}\left(\varepsilon^{2}\right)$. For smaller Mach numbers, 
the correct scaling is not visible as here round off errors caused by machine precision are dominating.

In order to enhance the influence of the complex eigenvalues of the explicit part we change the initialization of the velocity field of the low Mach vortex given in Eq. (4.1) to

$$
u^{0}(x)=\varepsilon u_{\infty}+\varepsilon\left(\begin{array}{c}
-x_{2} \\
x_{1}
\end{array}\right) \frac{e^{\frac{1}{2}\left(1-\|x\|_{2}^{2}\right)}}{2 \pi} .
$$

The resulting incompressible state changes to

$$
\rho_{(0)}=1, \quad \boldsymbol{u}_{(0)}=(0,0), \quad p_{(2)}=0 .
$$

With this initialization, we obtain complex eigenvalues at the cell boundaries if we choose $\boldsymbol{u}_{\infty} \neq 0$. We consider the case $\varepsilon=10^{-1}$ with $\boldsymbol{u}_{\infty}=(20,20)$ to investigate the effect of the potentially dominating complex eigenvalues and the influence of the modification on the solution on a grid with $16^{2}$ elements. For this testcase the scheme without modification fails at $t \approx 1$ whereas modified the scheme remains stable. As the Mach number of the dimensional equations of this case is $M \approx 2 \cdot 10^{-1}$ and the difference between the compressible and the incompressible solution is large, the solution obtained with the modification has to be considered concerning its validity. We compare the results at $t=10$ with the results obtained with an explicit $4^{\text {th }}$ order scheme. Considering the $L_{2}$-Errors in Table 1 shows for a reference Mach number of $\varepsilon=10^{-1}$ considerable modeling errors introduced by the modification which almost vanish for $\varepsilon=10^{-2}$. As in most low Mach number applications the focus of interest lies on the velocity field and not the acoustics, we compare the solution obtained with the modified scheme with the exact solution in Fig. 3. This reveals that the error introduced by the modification is not visible in the velocity field.

Table 1: $L_{2}$-Errors of vortex according to Eq. (4.2) at $t=10$ for $4^{\text {th }}$ order explicit DG scheme and modified RS-IMEX.

\begin{tabular}{||c|cccc||}
\hline$\varepsilon=10^{-1}$ & $L_{2}$-Error $(\rho)$ & $L_{2}$-Error $(\rho u)$ & $L_{2}$-Error $(\rho v)$ & $L_{2}$-Error $(E)$ \\
\hline explicit & $1.55 \cdot 10^{-6}$ & $1.49 \cdot 10^{-5}$ & $1.49 \cdot 10^{-5}$ & $3.99 \cdot 10^{-7}$ \\
RS-IMEX mod. & $2.69 \cdot 10^{-5}$ & $1.29 \cdot 10^{-4}$ & $1.29 \cdot 10^{-4}$ & $8.64 \cdot 10^{-6}$ \\
\hline$\varepsilon=10^{-2}$ & $L_{2}$-Error $(\rho)$ & $L_{2}$-Error $(\rho u)$ & $L_{2}$-Error $(\rho v)$ & $L_{2}$-Error $(E)$ \\
\hline explicit & $3.65 \cdot 10^{-8}$ & $6.38 \cdot 10^{-6}$ & $6.38 \cdot 10^{-6}$ & $1.84 \cdot 10^{-10}$ \\
RS-IMEX mod. & $4.90 \cdot 10^{-8}$ & $6.37 \cdot 10^{-6}$ & $6.37 \cdot 10^{-6}$ & $4.65 \cdot 10^{-9}$ \\
\hline
\end{tabular}

\subsection{Colliding pulses}

This testcase allows us to identify the influence of the modification on the solution and propagation velocities for different Mach numbers. Introduced by [26], it describes the 
exact solution

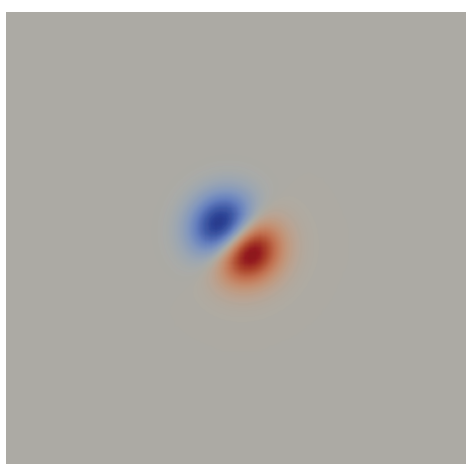

modified RS-IMEX

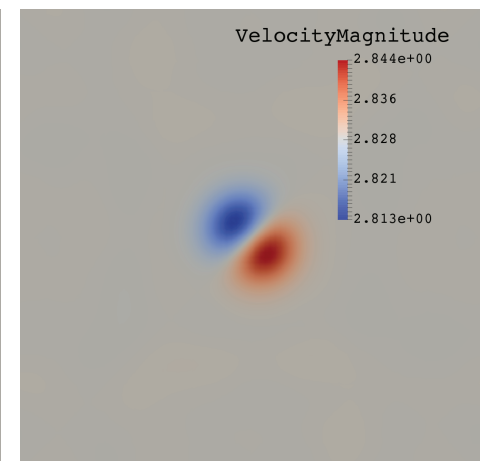

Figure 3: Velocity field at $t=10$ for low Mach vortex with $\varepsilon=10^{-1}$ (DG with $4^{\text {th }}$ order in space and time, $16^{2}$ elements)

one dimensional development of two colliding pulses at $\varepsilon=\frac{1}{11}$ on the domain $-L \leq x \leq$ $L=\frac{2}{\varepsilon}$. The initial data are given by

$$
\begin{aligned}
& \rho(x, t=0)=0.955+\varepsilon\left(1-\cos \left(\frac{2 \pi x}{L}\right)\right), \\
& u(x, t=0)=-\operatorname{sign}(x) \sqrt{\gamma}\left(1-\cos \left(\frac{2 \pi x}{L}\right)\right), \\
& p(x, t=0)=2 \gamma+\varepsilon \gamma\left(1-\cos \left(\frac{2 \pi x}{L}\right)\right),
\end{aligned}
$$

with $\gamma=1.4$. It is necessary to mention that this testcase, due to the $\varepsilon$-dependent domain, does not fit into the general framework; transforming it to a fixed domain would lead to another type of singularly perturbed equation than (2.1). However, treating it numerically is insightful to investigate to what extend the method presented here can be used.

The solution converges pointwise to the incompressible reference state

$$
\rho_{(0)}=0.955, \quad u_{(0)}=0, \quad p_{(2)}=0 .
$$

For the evaluation of this testcase we consider four different schemes:

1. A fully explicit scheme, which we consider to be the "true" solution;

2. the RS-IMEX scheme without modification, see Definition 2.1;

3. the RS-IMEX scheme with modification, see Definition 2.2;

4. and a fully implicit linear scheme neglecting the explicit flux in Definition 2.2 to show the influence of the nonlinear part. 

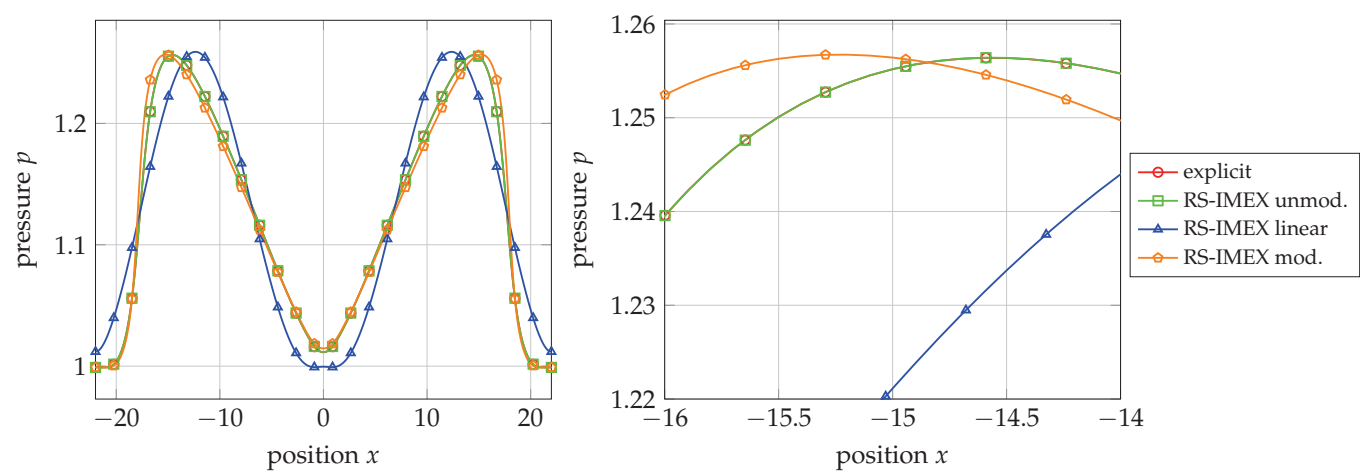

Figure 4: Pressure distribution at $t=1.63$ for colliding acoustic pulses with $\varepsilon=\frac{1}{11}$ calculated with different schemes (44 DG elements with $\mathcal{N}=7,3^{r d}$ order in time and $C F L=0.4$ ). Left: view of total domain; right: zoom to left pressure peak.
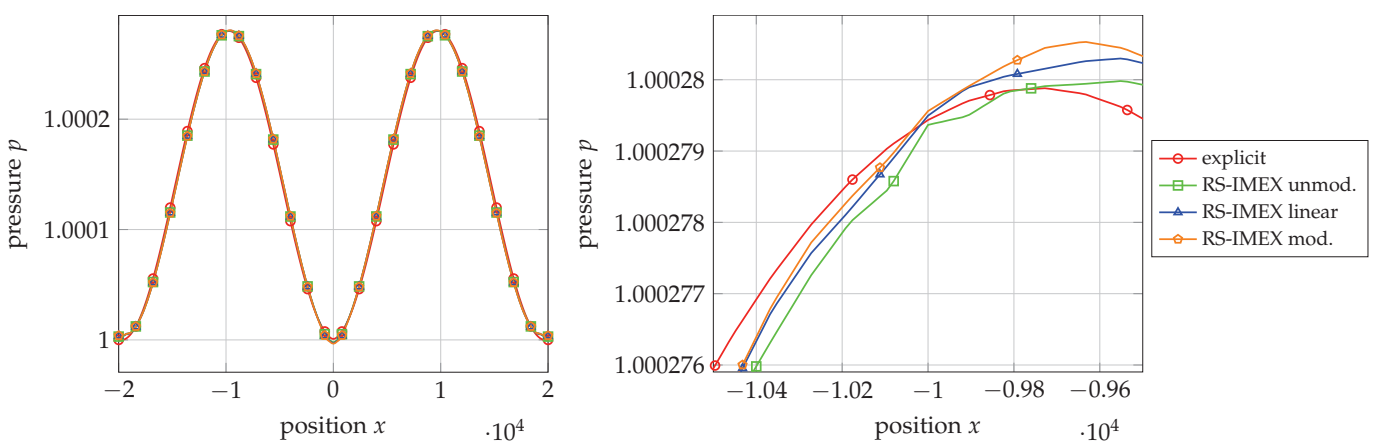

Figure 5: Pressure distribution at $t=1.63$ for colliding acoustic pulses with $\varepsilon=10^{-4}$ calculated with different schemes (44 DG elements with $\mathcal{N}=7,3^{\text {rd }}$ order in time and $\mathrm{CFL}=10^{-4}$ ). Left: view of total domain; right: zoom to left pressure peak.

Fig. 4 shows that for a moderately large Mach number $\left(\varepsilon=\frac{1}{11}\right)$, there are significant differences between the schemes. Whereas the RS-IMEX without any modification coincides with the explicit reference solution, the other schemes show different wave speeds. We see that the modified RS-IMEX scheme has qualitatively the same behavior as the full Euler equations but with a slightly faster propagation velocity. Neglecting the nonlinear terms results in other propagation velocities and no steepening of the pressure pulses. Hence, while the modified RS-IMEX scheme still shows qualitatively good results, the fully linear scheme is not applicable for such Mach numbers. Changing the reference Mach number to $\varepsilon=10^{-4}$ reveals that the influence of the nonlinear parts are very small. As displayed in Fig. 5 almost no differences between the schemes are visible. This illustrates the findings of Section 3.2 as the error introduced by neglecting (parts) of the explicit flux vanishes for $\varepsilon \rightarrow 0$. 


\subsection{Converging-diverging nozzle}

This testcase taken from [16] describes the two dimensional steady flow in a convergingdiverging nozzle. The incoming flow is accelerated by the nozzle and due to the outlet pressure a shock is formed in the diverging part of the nozzle. The occurring Mach numbers are not small and will illustrate the limitations of the introduced low Mach number scheme. The upper and lower nozzle contour being slip walls are given by $h^{ \pm}$

$$
h^{ \pm}(x)= \begin{cases} \pm 1, & \text { for }-2 \leq x<0 \\ \pm\left(\cos \left(\frac{\pi x}{2}\right)+3\right) / 4, & \text { for } 0 \leq x<4 \\ \pm 1, & \text { for } 4 \leq x \leq 8\end{cases}
$$

Analogous to [37] we prescribe the flux in normal direction on the slip walls as the velocity in this direction is zero

$$
\begin{aligned}
& \widetilde{\boldsymbol{f}}^{*}=\frac{\gamma-1}{\varepsilon^{2}}\left[E-\frac{\varepsilon^{2}}{2} \rho\left(\|\boldsymbol{u}\|_{2}^{2}-\left\|\boldsymbol{u}-\boldsymbol{u}_{\mathrm{ref}}\right\|_{2}^{2}\right)\right]\left(\begin{array}{lllll}
0 & n_{1} & n_{2} & n_{3} & 0
\end{array}\right)^{T}, \\
& \widehat{\boldsymbol{f}}^{*}=0, \\
& \boldsymbol{g}^{*}=p_{(2)}\left(\begin{array}{lllll}
0 & n_{1} & n_{2} & n_{3} & 0
\end{array}\right)^{T},
\end{aligned}
$$

with all variables being prescribed from the inner side. The initialization and the left inflow boundary condition at $-1 \leq y \leq 1$ is given by $\rho=1, u=0.355, v=0$ and $p=1$ corresponding to a Mach number of $M=0.3$. At the outflow the pressure $p=\frac{2}{3}$ is prescribed and the reference Mach number is chosen to be $\varepsilon=1$. For the spatial discretization we use a second order finite volume method with $400 \times 100$ elements and the second order IMEX-ARS-222 from [2] with $C F L=0.5$. Instead of using the numerical flux function as described in Eqs. (3.16) and (3.17) we use instead

$$
\widetilde{\Lambda}=\widehat{\Lambda}:=\max \left(\left|\boldsymbol{u}_{n}\right|+c\right),
$$

denoting the maximum local eigenvalue of the non-split system in face-normal direction with $c=\sqrt{\frac{\gamma p}{\rho}}$ being the local speed of sound. Fig. 6 shows that the unmodified RSIMEX scheme and the explicit scheme have similar solutions, whereas the position of the shock is slightly different for the modified RS-IMEX scheme. The position of the shock calculated with the explicit and the unmodified RS-IMEX scheme matches the results from [16]. The incompressible reference solution and the density field for the calculation with the unmodified FV RS-IMEX scheme are illustrated in Fig. 7.

Concluding, this testcase shows that the modified RS-IMEX splitting should not be applied to flows with high Mach numbers as the propagation velocities of the acoustic waves are not correct which leads to non-physical solutions. But we can see that also for a testcase where the incompressible reference solution shows a completely different flow field than the compressible one, the potentially complex eigenvalues might not cause trouble. 


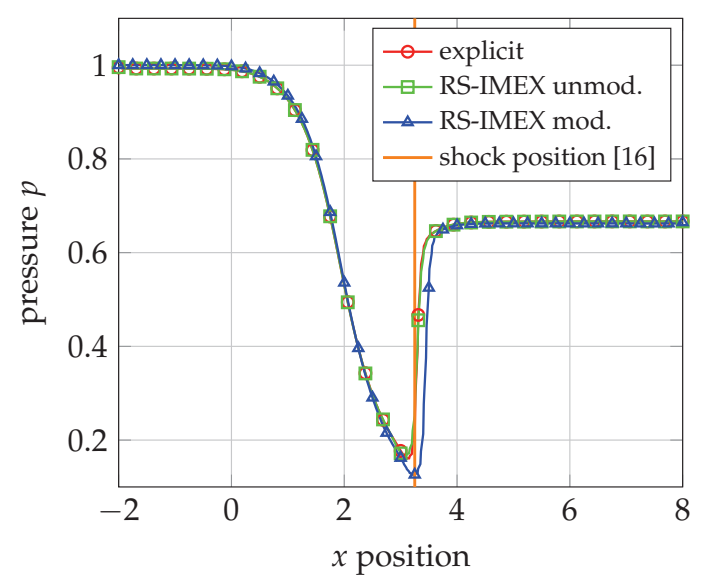

Figure 6: Pressure along $y=0.4$ calculated with explicit FV scheme and FV RS-IMEX with and without modification, indicated shock position according to [16]
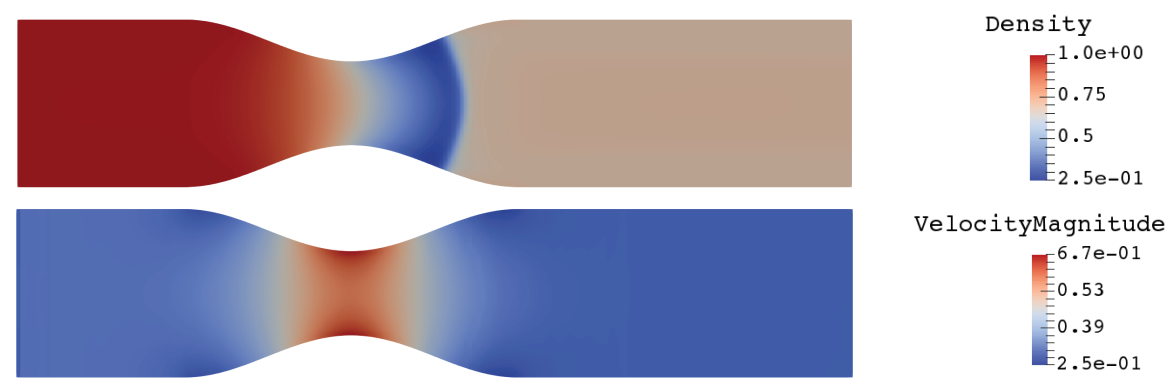

Figure 7: Density in converging-diverging nozzle at steady state, calculated with unmodified FV RS-IMEX (top). Velocity magnitude of incompressible reference solution after three time steps (bottom).

In this section, we have investigated the influence of the modification on the properties of the resulting scheme. We have shown that complex eigenvalues arising in the unmodified scheme can cause stability issues in some cases, but not in all. As this is not a priori clear for a specific testcase, our findings indicate that the modified scheme, which guarantees real eigenvalues, has to be used. We have further demonstrated that the introduced modeling error scales as expected with respect to $\varepsilon$, allowing the application of the scheme to low Mach number flow phenomena. This will be presented in the next section.

\section{$5 \quad$ Numerical experiments}

After having numerically analyzed the modeling error, we present in this section numerical results for flows at low Mach number, which is of course the natural habitat of this current method. 


\subsection{Gresho vortex}

The Gresho vortex describes a stationary rotating vortex and has been used to show the asymptotic preserving property in e.g. $[3,8,29]$. For an asymptotic preserving scheme, the vortex should remain unchanged regardless of the Mach number. The initialization of the Gresho vortex from [14] has been adapted to the nondimensional Euler equations to illustrate the properties of the RS-IMEX scheme. For the domain $[0,1]^{2}$ it is given by

$$
\begin{aligned}
& \rho=1, \quad \boldsymbol{u}=\boldsymbol{e}_{\phi} \begin{cases}5 r, & r<0.2, \\
2-5 r, & r<0.4, \\
0, & \text { else, }\end{cases} \\
& p= \begin{cases}p_{0}+\varepsilon^{2}\left(\frac{25}{2} r^{2}\right), & r<0.2, \\
p_{0}+\varepsilon^{2}\left(4 \ln (5 r)+4-20 r+\frac{25}{2} r^{2}\right), & r<0.4, \\
p_{0}+\varepsilon^{2}(4 \ln (2)-2), & \text { else, }\end{cases}
\end{aligned}
$$

with $p_{0}=0.5, r$ being the radius and $\boldsymbol{e}_{\phi}$ being the two dimensional azimuthal unit vector in polar coordinates. The transformation into cartesian coordinates is given by

$$
r=\sqrt{(x-0.5)^{2}+(y-0.5)^{2}} \quad \text { and } \quad \boldsymbol{e}_{\phi}=\left(\begin{array}{c}
-\sin (\arctan 2(y-0.5, x-0.5)) \\
\cos (\arctan 2(y-0.5, x-0.5))
\end{array}\right) .
$$

The hydrodynamic pressure is obtained by $p_{(2)}=\frac{1}{\varepsilon^{2}}\left(p-p_{0}\right)$. Fig. 8 shows the ratio of the local Mach number and the maximum Mach number at $\varepsilon=10^{-3}$ at different times. It is visible that the shape of the vortex remains almost unchanged. A comparison of the behavior at different reference Mach numbers $\varepsilon$ in Fig. 9 shows that $\varepsilon$ has no influence on the temporal evolution of the velocity field. Together with Fig. 8 this illustrates the low Mach number capabilities of the scheme.

\subsection{Double shear layer}

This testcase has been taken from [12] and has been studied more recently in [8]. It describes the incompressible flow of a developing shear layer on the two dimensional periodic domain $\Omega=[0,2 \pi]^{2}$. The initial data are given by

$$
\begin{aligned}
& \rho(x, y, t=0)=\frac{\pi}{15} \\
& u(x, y, t=0)=\left\{\begin{array}{ll}
\tanh \left(\left(y-\frac{\pi}{2}\right) / \rho\right), & y \leq \pi, \\
\tanh \left(\left(\frac{3 \pi}{2}-y\right) / \rho\right), & \text { else, }
\end{array} \quad v(x, y, t=0)=0.05 \sin (x) .\right.
\end{aligned}
$$

The pressure for the compressible calculation has been set to $p=1 / \gamma$ with $\gamma=1.4$, the hydrodynamic pressure has been initialized to $p_{(2)}=0$. We calculate this testcase with the DG scheme using $\mathcal{N}=5$ and $16^{2}$ elements as spatial and $3^{\text {rd }}$ order IMEX-ARS-443 

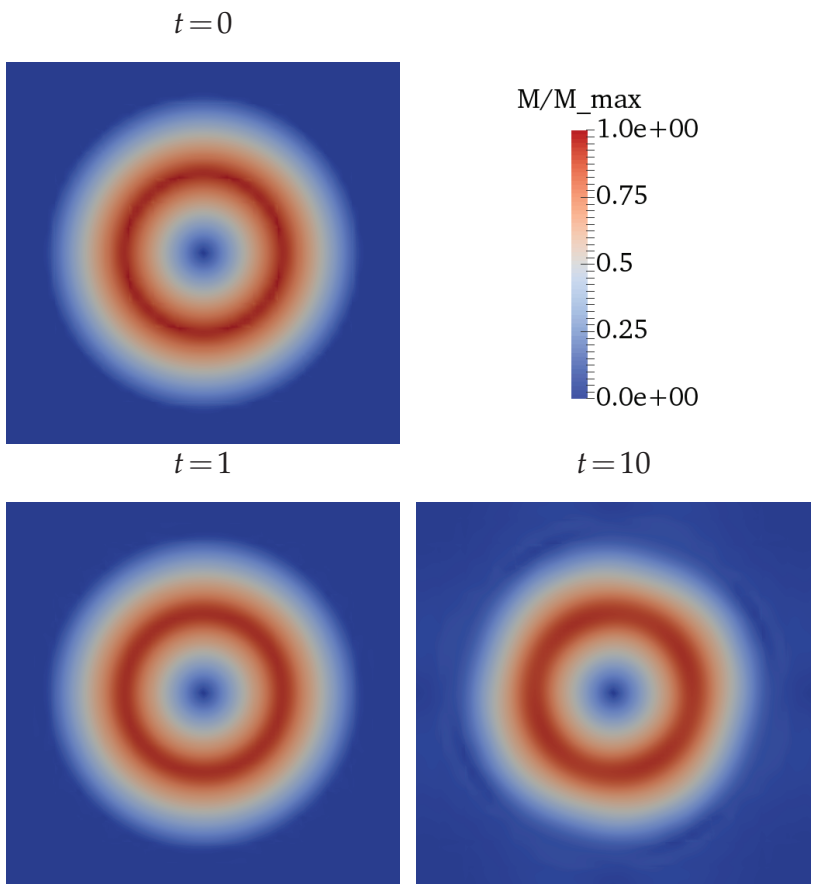

Figure 8: Relative Mach number of Gresho vortex for $\varepsilon=10^{-3}$ at different times calculated with the modified RS-IMEX ( $3^{\text {rd }}$ order in time and $4^{\text {th }}$ order in space with $16^{2}$ DG elements and CFL $=0.2$ ).

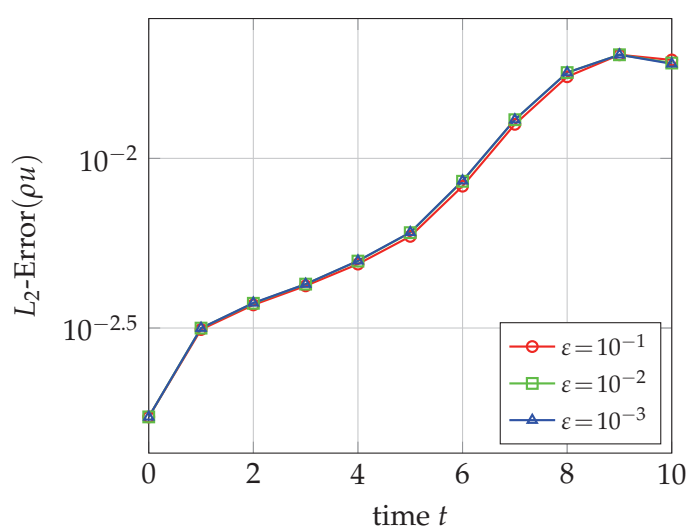

Figure 9: Temporal evolution of the $L_{2}$-error of the $x$-momentum for the Gresho vortex at different reference Mach numbers $\varepsilon$, computed with the modified RS-IMEX.

with $\mathrm{CFL}=0.5$ as temporal discretization. Considering the vorticity in Fig. 10 shows good agreement with the very accurate incompressible reference results from [8] obtained with a Fourier spectral code. Again, no dependency on the reference Mach number is visible. 

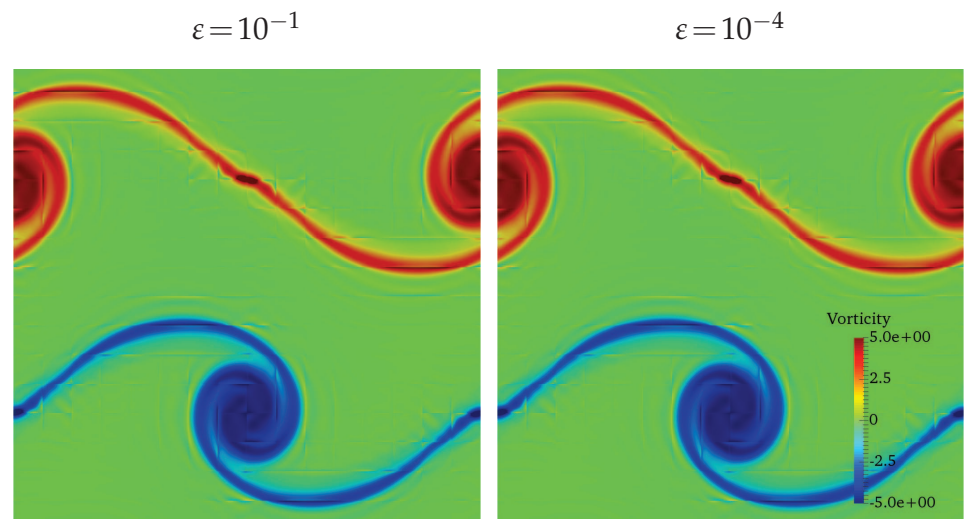

Figure 10: Vorticity of double shear layer calculated with the modified RS-IMEX for the reference Mach numbers $\varepsilon=10^{-1}$ (left) and $\varepsilon=10^{-4}$ (right) at final time $t=6$.

\subsection{Baroclinic vorticity generation problem}

This problem has been considered in [29] and describes the two dimensional interaction of an acoustic wave with a layered density. The compressible initial data with $\varepsilon=0.05$ on the periodic domain $-L \leq x \leq L:=\frac{1}{\varepsilon}, 0 \leq y \leq L_{y}:=\frac{2 L}{5}$ are given by

$$
\begin{aligned}
& \rho(x, y, t=0)=\rho_{0}+\frac{\varepsilon}{2000}\left(1+\cos \left(\frac{\pi x}{L}\right)\right)+\Phi(y), \\
& u(x, y, t=0)=\frac{1}{2} u_{0}\left(1+\cos \left(\frac{\pi x}{L}\right)\right), \\
& v(x, y, t=0)=0, \\
& p(x, y, t=0)=p_{0}+\frac{1}{2} \varepsilon \gamma\left(1+\cos \left(\frac{\pi x}{L}\right)\right),
\end{aligned}
$$

with $\rho_{0}=1, u_{0}=\sqrt{\gamma}, p_{0}=1, \gamma=1.4$ and

$$
\Phi(y)= \begin{cases}1.8 \frac{y}{L_{y}}, & \text { if } 0 \leq y \leq \frac{L_{y}}{2}, \\ 1.8\left(\frac{y}{L_{y}}-1\right), & \text { else. }\end{cases}
$$

The corresponding incompressible initialization is given by

$$
\begin{aligned}
& \rho_{(0)}(x, y, t=0)=\rho_{0}+\Phi(y), \quad u_{(0)}(x, y, t=0)=\frac{1}{2} u_{0}, \\
& v_{(0)}(x, y, t=0)=0, \quad p_{(2)}(x, y, t=0)=0 .
\end{aligned}
$$

The different accelerations caused by the acoustic wave in the two density layers lead to rotational excitation. Hence, a long wave sinusoidal shear layer is formed. After several passes of the acoustic wave, the sinusoidal shear layer becomes instable and smaller but 


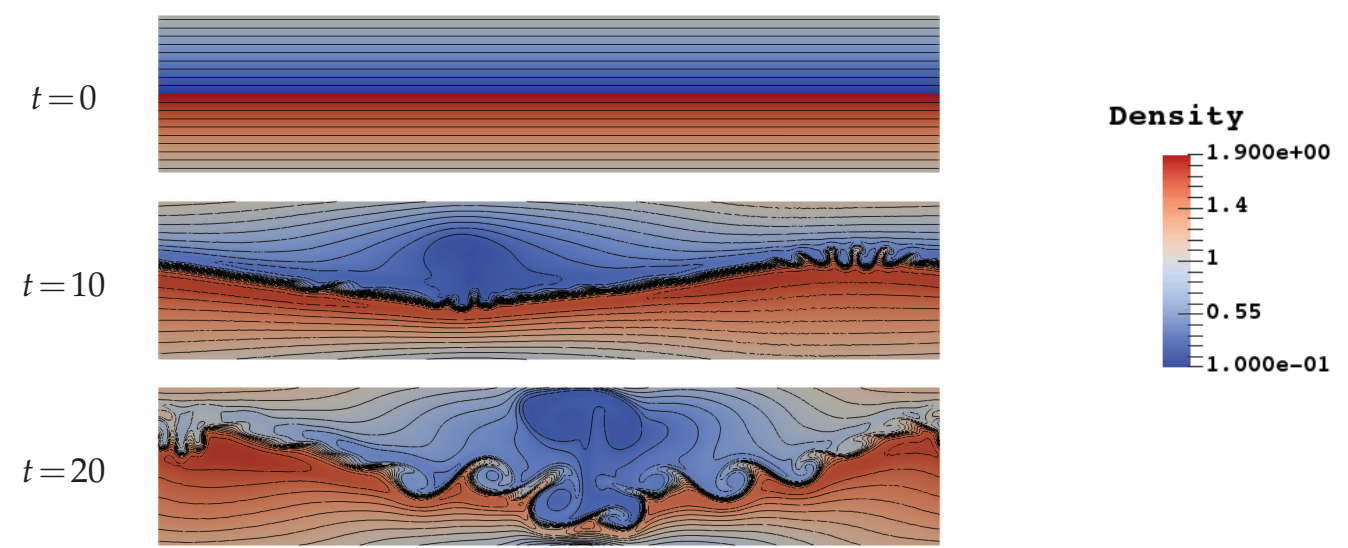

Figure 11: Density of barotropic vorticity generation problem at different times calculated with modified RSIMEX FV scheme with $2^{\text {nd }}$ order space and time.

fast growing vortices are generated. Caused by the density jump at $y=\frac{L_{y}}{2}$, the DG scheme (without limiting) is instable for this test case. Hence, we use a second order finite volume scheme with minmod limiter for the calculations with $800 \times 160$ elements and use IMEXARS-222 with CFL $=0.1$ for time integration.

Fig. 11 shows the temporal evolution of the instabilities. Whereas at $t=10$ the sinusoidal deformation of the shear layer is fully developed, its small scale disturbances are just starting to grow. At $t=20$ we see that the instabilities have grown considerably, forming Kelvin-Helmholtz vortices. The development of the instabilities is strongly dependent on the chosen numerical scheme and discretization parameters allowing only a qualitative comparison with the results from [29]. Considering the solution at the final times for the present scheme and for the scheme from [29], the density fields show a good agreement. Note that same flow patterns do not occur at the same simulation time for different schemes.

Note that for this testcase, the same reasoning as in Section 4.2 holds; due to the $\varepsilon$-dependent geometry, the underlying singularly perturbed equation is different to the one assumed. Still, it is worth noticing that the algorithm produces good results.

\subsection{Flow over a cylinder}

With this testcase the ability of using non-periodic boundary conditions as well as the asymptotic preserving property can be illustrated. We consider the two dimensional flow over a cylinder with slip walls and choose the initial data as

$$
\rho=1, \quad u=1, \quad v=0, \quad p=\frac{1}{\gamma},
$$

with $\gamma=1.4$ and the hydrodynamic pressure for the incompressible solver is $p_{(2)}=0$. At the inflow, a Dirichlet boundary condition with state given in Eq. (5.5) is used, whereas at 

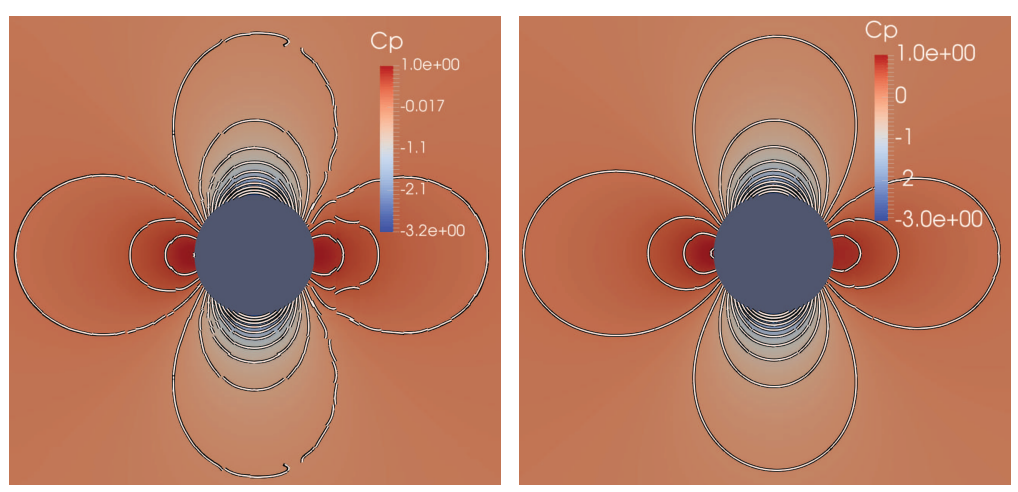

Figure 12: Isocontours and coloring of pressure coefficient at $\varepsilon=10^{-1}$ (left) and $\varepsilon=10^{-3}$ (right) calculated with the modified RS-IMEX.

the outflow only the momentum is prescribed. For the calculation we use a $3^{\text {rd }}$ order DG scheme and IMEX-ARS- 443 with $C F L=0.5$. To show the asymptotic preserving property if wall boundary conditions are present, we consider the pressure coefficient

$$
C_{p}=\frac{p-p_{\infty}}{\frac{1}{2} \varepsilon^{2} \rho_{\infty}\left\|u_{\infty}\right\|^{2}}
$$

where the pressure is calculated via the equation of state. For a vanishing Mach number, an exact solution is known (see e.g. [1]) from potential flow where $C_{p}$ ranges from -3 to 1 . In Fig. 12 the pressure coefficient is displayed for the reference Mach numbers $\varepsilon=10^{-1}$ and $10^{-3}$ showing the flow characteristics of potential flow. As the pattern of the isocontours and the numerical range of the pressure coefficient is almost the same for both Mach numbers, we have evidence that the present scheme is asymptotic preserving also if wall boundary conditions are present.

\subsection{Taylor-Green vortex}

As a final testcase we consider the tree dimensional Taylor-Green vortex which has been used to investigate the dissipative behavior of numerical schemes at low Mach numbers for inviscid Euler equations in [3] and [37]. The incompressible initialization of the Taylor-Green vortex on a periodic box with dimensions $\Omega=[0,2 \pi]^{3}$ is given by

$$
\begin{aligned}
& \rho_{(0)}=1, \\
& \boldsymbol{u}_{(0)}(x, t=0)=V_{0}\left(\begin{array}{c}
\cos \left(x_{1}\right) \cos \left(x_{2}\right) \cos \left(x_{3}\right) \\
-\cos \left(x_{1}\right) \sin \left(x_{2}\right) \cos \left(x_{3}\right) \\
0
\end{array}\right), \\
& p_{(2)}(x, t=0)=\frac{\rho_{(0)} V_{0}^{2}}{16}\left(\cos \left(2 x_{1}\right)+\cos \left(2 x_{2}\right)\right)\left(\cos \left(2 x_{3}\right)+2\right),
\end{aligned}
$$




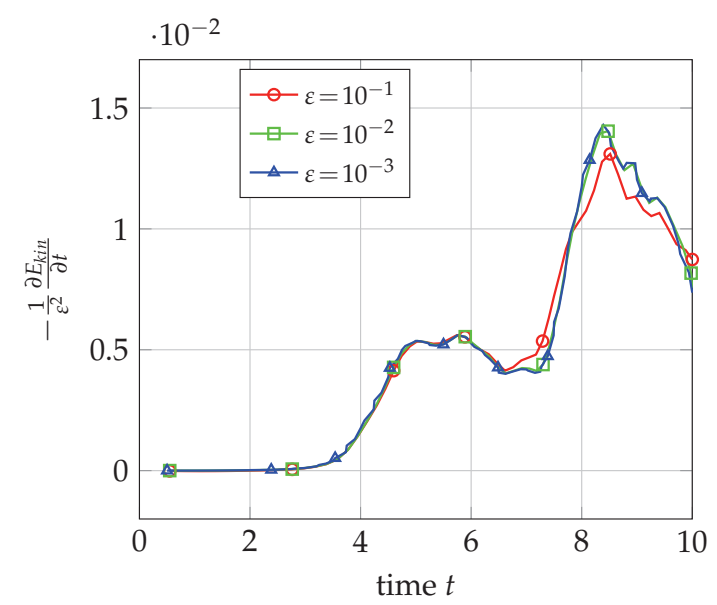

Figure 13: Scaled change rate of kinetic energy for TGV at different reference Mach numbers $\varepsilon$ (DG scheme with $3^{\text {rd }}$ order in space and time, CFL $=0.9$ and $16^{3}$ elements, modified RS-IMEX).

where $V_{0}$ denotes a constant initial velocity which is chosen to be $V_{0}=1 ; x_{1}, x_{2}$ and $x_{3}$ denote the spatial coordinates. The initialization for the compressible solver reads

$$
\begin{aligned}
& \rho(x, t=0)=1 \\
& u(x, t=0)=V_{0}\left(\begin{array}{c}
\cos \left(x_{1}\right) \cos \left(x_{2}\right) \cos \left(x_{3}\right) \\
-\cos \left(x_{1}\right) \sin \left(x_{2}\right) \cos \left(x_{3}\right) \\
0
\end{array}\right), \\
& p(x, t=0)=\frac{\rho V_{0}^{2}}{\gamma}+\frac{\rho V_{0}^{2} \varepsilon^{2}}{16}\left(\cos \left(2 x_{1}\right)+\cos \left(2 x_{2}\right)\right)\left(\cos \left(2 x_{3}\right)+2\right),
\end{aligned}
$$

with $\gamma=1.4$ resulting in a maximum Mach number of $\varepsilon$. We consider the scaled change rate of the kinetic energy defined as

$$
\frac{\partial E_{k i n}}{\varepsilon^{2} \partial t}:=\frac{\partial}{\partial t}\left(\frac{1}{2} \rho\|\boldsymbol{u}\|_{2}^{2}\right)
$$

as an indicator of the dissipative characteristic. For an asymptotic consistent scheme, the scaled change rate of the kinetic energy should be the same for different reference Mach numbers as displayed in Fig. 13. Slight variations are only visible for $\varepsilon=10^{-1}$ probably caused by compressibility as already observed in [37] thereby confirming the AP property of the scheme also for this $3 \mathrm{~d}$ case.

\section{Conclusion and outlook}

In this work, we have presented an IMEX splitting of the full Euler equations based on the incompressible reference solution. We have shown that it is (semi-discrete in time) 
asymptotically consistent. Numerical experiments have demonstrated that this modified RS-IMEX scheme avoids stability problems induced by complex eigenvalues and offers a possibility to capture the convective phenomena correctly in the case of low Mach numbers. We have also seen that it is not suitable for large Mach numbers, which is not a surprise as its construction rational relies on introducing a modeling error of $\mathcal{O}\left(\varepsilon^{2}\right)$. Furthermore, we have seen that the straightforward application of the RS-IMEX, so without a modification, gives rise to complex eigenvalues. In many applications, though, this does not seem to be a problem which is subject of current investigations.

The developed scheme is potentially attractive for efficient computations of low Mach number flows as it is high order in space and time and requires no implicit solution of a nonlinear system other than a solve for the incompressible solution. Due to the relatively large zoo of different splittings (and other low-Mach schemes), the most eminent question is to compare these. This is currently work in progress. In some sense, the contributions in this paper are very relevant for this, because now also the RS-IMEX can be compared against other schemes. Last but not least, we consider a pure low Mach scheme as an intermediate step only. The long-term goal is the construction of a suitable all-Mach scheme.

\section{Acknowledgments}

K. Kaiser has been partially supported by the German Research Foundation (DFG) through project NO 361/6-1; his study was supported by the Special Research Fund (BOF) of Hasselt University. M. Lukáčová has been partially supported by the TRR 165 "Waves to weather". J. Zeifang has been supported by the German Research Foundation (DFG) through the International Research Training Group GRK 2160/1: Droplet Interaction Technologies. We acknowledge the support and the computing time provided by the High Performance Computing Center Stuttgart (HLRS) through the hpcdg project.

\section{References}

[1] J. D. Anderson. Fundamentals of Aerodynamics. McGraw Hill New York, 2001.

[2] U. M. Ascher, S. Ruuth, and R. Spiteri. Implicit-explicit Runge-Kutta methods for timedependent partial differential equations. Applied Numerical Mathematics, 25:151-167,1997.

[3] W. Barsukow, P. V. F. Edelmann, C. Klingenberg, F. Miczek, and F. K. Röpke. A numerical scheme for the compressible low-Mach number regime of ideal fluid dynamics. Journal of Scientific Computing, 72(2):623-646, 2017.

[4] G. Bispen, K. R. Arun, M. Lukáčová-Medvid'ová, and S. Noelle. IMEX large time step finite volume methods for low Froude number shallow water flows. Communications in Computational Physics, 16:307-347, 2014.

[5] G. Bispen, M. Lukáčová-Medvid'ová, and L. Yelash. Asymptotic preserving IMEX finite volume schemes for low Mach number Euler equations with gravitation. Journal of Computational Physics, 335:222-248, 2017. 
[6] S. Boscarino. Error analysis of IMEX Runge-Kutta methods derived from differentialalgebraic systems. SIAM Journal on Numerical Analysis, 45:1600-1621, 2007.

[7] S. Boscarino, L. Pareschi, and G. Russo. Implicit-explicit Runge-Kutta schemes for hyperbolic systems and kinetic equations in the diffusion limit. SIAM Journal on Scientific Computing, 35(1):A22-A51, 2013.

[8] S. Boscarino, G. Russo, and L. Scandurra. All Mach number second order semi-implicit scheme for the Euler equations of gasdynamics. Journal of Scientific Computing, 77:850$884,2018$.

[9] F. Cordier, P. Degond, and A. Kumbaro. An asymptotic-preserving all-speed scheme for the Euler and Navier-Stokes equations. Journal of Computational Physics, 231:5685-5704, 2012.

[10] P. Degond, S. Jin, and J.-G. Liu. Mach-number uniform asymptotic-preserving gauge schemes for compressible flows. Bulletin-Institute of Mathematics Academia Sinica (New Series), 2(4):851-892, 2007.

[11] M. Dumbser and V. Casulli. A conservative, weakly nonlinear semi-implicit finite volume scheme for the compressible Navier-Stokes equations with general equation of state. Applied Mathematics and Computation, 272:479-497, 2016.

[12] W. E and C.-W. Shu. A numerical resolution study of high order essentially non-oscillatory schemes applied to incompressible flow. Journal of Computational Physics, 110(1):39-46, 1994.

[13] D. Ghosh and E. M. Constantinescu. Semi-implicit time integration of atmospheric flows with characteristic-based flux partitioning. SIAM Journal on Scientific Computing, 38(3):A1848-A1875, 2016.

[14] P. M. Gresho and S. T. Chan. On the theory of semi-implicit projection methods for viscous incompressible flow and its implementation via a finite element method that also introduces a nearly consistent mass matrix. Part 2: Implementation. International Journal for Numerical Methods in Fluids, 11(5):621-659, 1990.

[15] J. Haack, S. Jin, and J.-G. Liu. An all-speed asymptotic-preserving method for the isentropic Euler and Navier-Stokes equations. Communications in Computational Physics, 12:955-980, 2012.

[16] R. Hartmann and P. Houston. Adaptive discontinuous Galerkin Finite Element methods for the compressible Euler Equations. Journal of Computational Physics, 183:508-532, 2002.

[17] F. Hindenlang, G. Gassner, C. Altmann, A. Beck, M. Staudenmaier, and C.-D. Munz. Explicit discontinuous Galerkin methods for unsteady problems. Computers \& Fluids, 61:86-93, 2012.

[18] W. Hundsdorfer and S.-J. Ruuth. IMEX extensions of linear multistep methods with general monotonicity and boundedness properties. Journal of Computational Physics, 225(2):2016$2042,2007$.

[19] P. Jin, X. Deng, and F. Xiao. A direct ale multi-moment finite volume scheme for the compressible euler equations. Communications in Computational Physics, 24:1300-1325, 2018.

[20] S. Jin. Asymptotic preserving (AP) schemes for multiscale kinetic and hyperbolic equations: A review. Rivista di Matematica della Universita Parma, 3:177-216, 2012.

[21] K. Kaiser and J. Schütz. A high-order method for weakly compressible flows. Communications in Computational Physics, 22(4):1150-1174, 2017.

[22] K. Kaiser, J. Schütz, R. Schöbel, and S. Noelle. A new stable splitting for the isentropic Euler equations. Journal of Scientific Computing, 70:1390-1407, 2017.

[23] K. Kaiser, J. Zeifang, J. Schütz, A. Beck, and C.-D. Munz. Comparison of different splitting techniques for the isentropic Euler equations. IGPM Preprint 476 / CMAT Preprint 
UP-18-01, 2018.

[24] C. A. Kennedy and M. H. Carpenter. Additive Runge-Kutta schemes for convectiondiffusion-reaction equations. Applied Numerical Mathematics, 44:139-181, 2003.

[25] S. Klainerman and A. Majda. Singular limits of quasilinear hyperbolic systems with large parameters and the incompressible limit of compressible fluids. Communications on Pure and Applied Mathematics, 34:481-524, 1981.

[26] R. Klein. Semi-implicit extension of a Godunov-type scheme based on low Mach number asymptotics I: One-dimensional flow. Journal of Computational Physics, 121:213-237, 1995.

[27] D. A. Kopriva. Implementing Spectral Methods for Partial Differential Equations: Algorithms for Scientists and Engineers. Springer Publishing Company Incorporated, 1st edition, 2009.

[28] H. Liu and J. Zou. Some new additive Runge-Kutta methods and their applications. Journal of Computational and Applied Mathematics, 190(1-2):74-98, 2006.

[29] S. Noelle, G. Bispen, K. R. Arun, M. Lukáčová-Medvid'ová, and C.-D. Munz. A weakly asymptotic preserving low Mach number scheme for the Euler equations of gas dynamics. SIAM Journal on Scientific Computing, 36:B989-B1024, 2014.

[30] J. Qi, B. Tian, and J. Li. A high-resolution cell-centered lagrangian method with a vorticity-based adaptive nodal solver for two-dimensional compressible euler equations. Communications in Computational Physics, 24:774-790, 2018.

[31] P. L. Roe. Characteristic-based schemes for the Euler equations. Annual review of fluid mechanics, 18(1):337-365, 1986.

[32] S. Schochet. The compressible Euler equations in a bounded domain: existence of solutions and the incompressible limit. Communications in Mathematical Physics, 104(1):49-75, 1986.

[33] M. Sonntag and C.-D. Munz. Efficient parallelization of a shock capturing for discontinuous Galerkin methods using finite volume sub-cells. Journal of Scientific Computing, 70(3):1262$1289,2017$.

[34] M. Tavelli and M. Dumbser. A pressure-based semi-implicit space-time discontinuous Galerkin method on staggered unstructured meshes for the solution of the compressible Navier-Stokes equations at all Mach numbers. Journal of Computational Physics, 341:341376, 2017.

[35] E. Turkel. Preconditioned methods for solving the incompressible and low speed compressible equations. Journal of Computational Physics, 72(2):277 - 298, 1987.

[36] H. Zakerzadeh. Asymptotic analysis of the RS-IMEX scheme for the shallow water equations in one space dimension. IGPM Preprint Nr. 455, 2016.

[37] J. Zeifang, K. Kaiser, A. Beck, J. Schütz, and C.-D. Munz. Efficient high-order discontinuous Galerkin computations of low Mach number flows. Communications in Applied Mathematics and Computational Science, 13(2):243-270, 2018. 